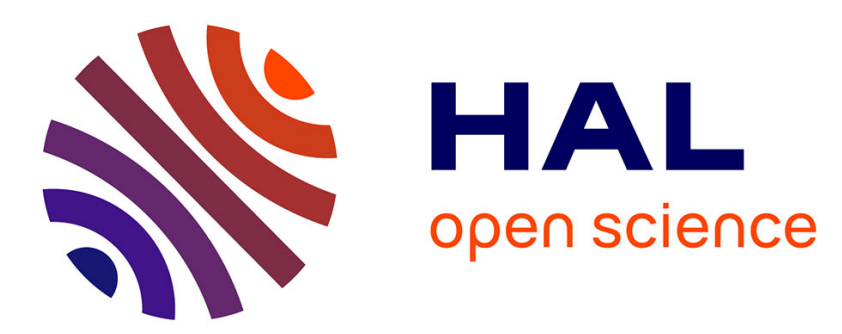

\title{
Services publics et fractures de la ville. La " pensée publique " entre diversité, éclatement et souci du rapprochement
}

Thomas Kirszbaum

\section{To cite this version:}

Thomas Kirszbaum. Services publics et fractures de la ville. La " pensée publique " entre diversité, éclatement et souci du rapprochement . Sociologie du Travail, 2004, pp.224-260. halshs-01100685

\section{HAL Id: halshs-01100685 \\ https://shs.hal.science/halshs-01100685}

Submitted on 6 Jan 2015

HAL is a multi-disciplinary open access archive for the deposit and dissemination of scientific research documents, whether they are published or not. The documents may come from teaching and research institutions in France or abroad, or from public or private research centers.
L'archive ouverte pluridisciplinaire HAL, est destinée au dépôt et à la diffusion de documents scientifiques de niveau recherche, publiés ou non, émanant des établissements d'enseignement et de recherche français ou étrangers, des laboratoires publics ou privés. 


\title{
Services publics et fractures de la ville \\ La « pensée publique » entre diversité, éclatement \\ et souci du rapprochement $\widehat{\imath}$
}

\author{
Thomas Kirszbaum
}

Sociologie du travail 46 (2), 2004, 224-260

Évoquer une «pensée publique » à propos des relations entre les services publics et la ville ne va pas de soi. D'abord parce que la sphère publique n'a pas un cerveau unique, mais dispose de multiples instances chargées, avec l'appui d'experts, de produire des analyses de nature à guider son action. $\mathrm{Si}$ « pensée » il y a, elle s'incarne dans un corpus hétérogène ; rapports officiels commandités à de hauts fonctionnaires, universitaires ou personnalités qualifiées; recherches effectuées en réponse à des appels d'offres publics ; revues spécialisées, ouvrages collectifs, colloques et séminaires sponsorisés par des administrations ou des organismes au statut variable (Caisse des dépôts et consignations, Union nationale des fédérations HLM, Conseil national de la fonction publique territoriale, etc.). Parmi les acteurs publics les plus investis dans la réflexion sur les services publics et la ville, quatre jouent un rôle prépondérant ; le Puca (Plan urbain construction architecture) $^{1}$ et la Drast (Direction de la recherche et des affaires scientifiques et techniques), tous deux rattachés au ministère de l'Équipement; la DIV (Délégation interministérielle à la ville) et la RATP22

\footnotetext{
Ce texte a fait l'objet d'une lecture attentive par Dominique Lorrain, remercié ici pour ses stimulantes observations.

${ }^{1}$ Le Puca a repris, à partir de 1999, les missions du Plan urbain et du Plan construction et architecture.

${ }^{2}$ De façon périodique, les administrations éprouvent le besoin de récapituler et synthétiser les acquis du champ d'expertises qu'elles contribuent à produire. Cet article prend justement appui, pour partie, sur des matériaux rassemblés à l'occasion d'une synthèse bibliographique de ce type, commanditée par le Puca (Kirszbaum, 2001).
} 
Il peut sembler également excessif de parler d'une «pensée publique » à propos d'objets aussi hétérogènes que sont, respectivement, les services publics et l'urbain. Les premiers regroupent un ensemble d'activités et de modes de gestion disparates, selon qu'il s'agit d'entreprises nationales ou de services publics locaux organisés en réseaux, de services déconcentrés de l'État ou de services des collectivités locales, du secteur HLM ou d'associations exerçant de fait des missions de service public, selon aussi qu'ils délivrent des services aux personnes ou s'adressent à des entités collectives (administrations, collectivités, entreprises). L'urbain désigne quant à lui des modalités contrastées d'habiter la ville et la frontière avec le rural se fait de plus en plus poreuse. Les objets « services publics » et «ville» renvoient ainsi à des réalités multiformes, investies par des discours sectoriels qui se prêtent mal à la généralisation.

Cet article en forme de «review» examine les liens entre les expertises scientifiques et les priorités publiques, à commencer par celles de l'État. Le corpus étudié comprend une majorité de documents publiés entre 1990 et 2002, période durant laquelle le discours public semble se chercher face aux mutations simultanées des services publics et de la ville, voire leur crise. Cette délimitation temporelle n'interdit pas de faire retour sur des textes plus anciens dans la mesure où ils préfigurent des orientations futures ou s'ils témoignent d'une forme « d'amnésie » des productions contemporaines.

L'exhaustivité étant hors d'atteinte, le corpus rassemblé met en relief des textes représentatifs d'une doctrine publique ou de positions scientifiques dominantes, les deux sphères étant loin d'être hermétiques l'une à l'autre. Ces textes ont été répertoriés selon leur caractère plus ou moins officiel ; la littérature grise, de type « rapport officiel », a directement vocation à fournir une doctrine aux pouvoirs publics ; le financement de recherches, séminaires et colloques par des administrations renseigne surtout sur la nature des préoccupations publiques à une période donnée ; enfin, les résultats des recherches, qu'ils soient publiés à l'initiative des chercheurs ou que ces derniers bénéficient d'une tribune publique (dans une revue, un séminaire, un colloque...), doivent être évoqués car ils font parfois l'objet d'une appropriation officielle, même tardive, par les pouvoirs 
publics. Sur des objets tels que les services publics et de la ville, le mouvement des idées est en partie stimulé par la commande publique et les décideurs s'approprient ces idées dès lors qu'elles s'accordent à leurs priorités du moment.

Un second principe de classification du corpus a trait à son contenu. La relation des services publics et de la ville peut être appréhendée sous trois angles ; l'offre territoriale des services, leurs rapports avec l'environnement et les normes permettant de penser et justifier les mutations à l'œuvre. Quelle que soit la dimension privilégiée, trois registres de discours sont identifiables autour des figures suivantes; la diversité, l'éclatement et le rapprochement. C'est sous le signe de la diversité que sont pensées, à la fois, l'adaptation de l'offre de services aux singularités des territoires et de leurs habitants, les formules de coordination entre les services et leur environnement, ainsi que les évolutions normatives conduisant à «territorialiser» l'intérêt général. Une autre lecture des évolutions en cours insiste davantage sur la crise des services publics dans un contexte de spécialisation sociale et ethnique des territoires, ce dont rend compte l'image de la «ville éclatée» (May et al., 1998). Plutôt qu'aux notions de choix, de coordination et d'intérêt général local, ce registre de discours se réfère aux thèmes de la ségrégation, du conflit et du déclin de la norme d'universalité des services publics. Face aux quartiers pauvres, et dans un contexte d'éclatement de la ville comme de l'action publique elle-même (Lefèvre, 1998), la priorité des pouvoirs publics est au « rapprochement». Cette stratégie se décline comme une exigence de proximité ; elle prend corps dans des dispositifs de médiation appelés à résorber la conflictualité entre les services et les habitants des quartiers pauvres ; le rapprochement trouve enfin sa justification normative dans l'invocation d'un « droit commun ». Les pouvoirs publics semblent avoir pensé, depuis une dizaine d'années, la nécessité du rapprochement comme le remède à une «cohésion sociale et territoriale» menacée par l'éclatement de la ville et des services publics. Ce souci du rapprochement, qu'expriment notamment les objectifs de la politique de la ville, n'est pourtant pas sans équivoques. 


\section{Les services publics sous le signe de la diversité}

\subsection{L'ouverture des choix}

On qualifie parfois de «service public à la française», un modèle historiquement fondé sur la production monopolistique et standardisée de certains services organisés en réseaux (poste, chemin de fer, électricité...) que la puissance publique a pris en charge, afin de répondre aux besoins d'équipement nés de l'urbanisation du pays. Ce modèle est apparu menacé, à la fin des années 1980, par l'intégration européenne qui impose la mise en concurrence progressive des secteurs public et privé. Quand ce thème émerge sur l'agenda public français, la haute administration cherche à se forger une doctrine nouvelle, en appui sur des groupes de travail et publications où grands commis de l'État et chercheurs mêlent leurs réflexions (Annales des mines, réalités industrielles, 1991 ; Conseil d'État, 1994 ; Stoffaës, 1995 ; Denoix de Saint Marc, 1996 ; Bergougnoux 2000). Ces travaux invitent les entreprises nationales à adopter une posture offensive pour s'adapter au nouveau contexte économique, technologique et sociétal dont l'Europe ne serait finalement que le révélateur. Les effets spatiaux de cette adaptation sous contrainte ont le plus souvent été débattus en comparant ses potentialités et périls pour un «modèle français » d'aménagement du territoire fondé, en principe, sur l'action redistributive des réseaux nationaux. Cette préoccupation, exprimée notamment par le groupe de travail du Commissariat général du plan dirigé par Christian Stoffaës (Stoffaës, 1995), n'a pas tardé à diffuser dans les sphères de réflexion du ministère de l'Équipement (Jeannot et Quin, 1997).

Dans les deux cas, et pour dédramatiser les effets territoriaux de l'ouverture à la concurrence, la réflexion publique a semblé (re)découvrir l'existence d'un « second modèle » de service public « à la française », celui des services urbains (eau, transports, chauffage, traitement des déchets, etc.) développés de longue date par l'alliance entre municipalités et grands groupes privés. Des chercheurs avaient montré la capacité de cette offre non monopolistique à concilier 
impératifs de rentabilité et missions de service public (Auby, 1982 ; Lorrain, 1990). À l'instar des textes doctrinaux redéfinissant le «modèle français » de service public en y intégrant les services urbains, le ministère de l'Équipement en a fait l'un de ses thèmes de recherche privilégiés (Martinand, 1993 ; DAEI, 1994 ; Plan urbain, 1995). Les partenariats public-privé sont désormais affichés par le ministère comme un élément clé du développement des services collectifs (Chatelus et Perrot, 2000).

Deux évolutions conjointes - l'ouverture à la concurrence des entreprises nationales et l'amplification, consécutive aux lois de décentralisation, des délégations municipales au secteur privé - dessinent le paysage plus diversifié d'une offre de services tenue de s'adapter aux spécificités locales et à celles des usagers. Les chercheurs sont néanmoins plus prompts que les rapports officiels à invoquer, pour caractériser ces évolutions, les concepts de marché (Chauvière et Godbout, 1992), de marketing (Hatchuel et al., 1993) ou de clientèle (Quatrebarbes, 1996). Jusqu'à quel point ces concepts pénètrent-ils la réflexion sur la ville? En dehors du champ des services urbains où le processus de privatisation ne fait guère de doute (Lorrain et Stoker, 1995), les thèmes du partage public-privé et de l'élargissement de la gamme des choix offerts aux usagers-consommateurs s'imposent plus tardivement comme les éléments d'une nouvelle «théorisation» de la ville opérée par des chercheurs régulièrement associés au ministère de l'Équipement. Encore cette lecture de la diversification des services dans la ville n'est-elle pas l'apanage de ce ministère. On la trouve à l'œuvre, par exemple, dans les secteurs de l'éducation et de la sécurité, où la dialectique des discours savants et officiels suit sa dynamique propre. Depuis les travaux pionniers de René Ballion (Ballion, 1982) sur les «consommateurs d'école » et ceux de Gabriel Langouët et Alain Léger (Langouët et Léger 1987) sur le «zapping d'école», de nombreuses recherches ont mis en évidence la tendance des usagers à jouer des complémentarités entre secteurs public et privé et à maximiser leurs choix au sein d'une offre publique différenciée. Ces travaux ont mis en lumière la rencontre entre la logique individualiste des usagers et la différenciation croissante de l'offre locale d'éducation, y compris celle des 
collectivités locales (cf. par exemple Thin et al., 1992 ; et, pour une synthèse des connaissances, Meuret et al., 2000). Pour décrire cette évolution, le rapport sur le collège de l'an 2000, confié à François Dubet (Dubet, 1999), s'est référé au concept de «marché scolaire », couramment avancé par les sociologues à partir des années 1990. Avec le rapport Hébrard sur la mixité scolaire (Hébrard, 2002), l'institution finit par faire sienne cette analyse, après avoir réalisé une enquête d'envergure pour prendre la mesure des phénomènes de «défection » hors sectorisation scolaire ou vers le privé (Chausseron, 2001).

La lecture des Cahiers de la sécurité intérieure éclaire les préoccupations du ministère de l'Intérieur au cours des années 1990 ; les numéros de la revue intitulés «L'offre publique de sécurité » $\left(1990, \mathrm{n}^{\circ} 2\right)$ «Le marché de la sécurité privée » $\left(1991, \mathrm{n}^{\circ} 3\right)$, «Collectivités locales et sécurité » $\left(1994, \mathrm{n}^{\circ} 16\right)$, «Les partages de la sécurité » $\left(1998, \mathrm{n}^{\circ} 33\right)$, sont révélateurs de l'importance croissante du fait urbain qui apparaît comme l'aiguillon d'une recomposition de l'offre locale de sécurité. Entre-temps, les sociétés privées ont acquis le statut de « coproducteurs » (Loi d'orientation et de programmation sur la sécurité du 21 janvier 1995) et les contrats locaux de sécurité, annoncés en 1997, font des maires les acteurs à part entière des politiques locales de sécurité. Celles-ci obéiraient de plus en plus à une logique de la demande, les élus étant les principaux relais des habitants. La connaissance de la demande est d'ailleurs l'un des buts recherchés par les Contrats locaux de sécurité (Ihesi, 1998).

Quelle place tiennent le marché et l'individu dans les réflexions du ministère de l'Équipement sur les services publics et la ville ? La dimension marchande a toujours existé dans le logement (Topalov, 1987) ou les transports (Lefèvre et Offner, 1990) qui comptent parmi ses principaux domaines de compétence. Depuis les années 1970, au moment où la crise des transports collectifs devient patente en milieu urbain et à partir de la réforme du financement du logement de 1977, l'enjeu central devient celui de la perte des parts de marché de l'offre publique - concédée ou non à des opérateurs privés - face à la montée en puissance de l'automobile et de l'accession à la propriété. D'innombrables études et séminaires établissent ces constats (cf. par exemple (Duhem et al., 1995) à 
propos des transports ; (Ascher, 1995) à propos du logement), en même temps qu'ils se penchent sur les déterminants individuels de la mobilité urbaine (Bieber et al., 1993 ; Wiel et al., 1997) ou résidentielle (Bonvalet et Fribourg, 1990). Dans ces deux secteurs, la réponse politique cherche de nouvelles lignes de partage entre offres publiques et privées, fondées sur leur complémentarité plutôt que leur opposition (cf. (Brun et Faivre d'Arcier, 1993) à propos des transports ; (Landrieu, 1997) à propos du logement).

Mais il faut attendre la seconde moitié de la décennie 1990 pour que ces diverses analyses sectorielles viennent étayer une reformulation des enjeux urbains, conduite par des chercheurs liés au ministère de l'Équipement, autour des figures du marché et de l'individu (Ascher, 1995; Ascher, 1998; Chalas et Dubois-Taine, 1997 ; May et al., 1998). L'évolution de la ville devient alors un argument pour la réforme des services publics. Conçue à l'origine pour traiter des cas standards selon une approche calquée sur l'organisation taylorienne du travail, ils devraient se faire à présent plus plastiques, proposer des prestations flexibles, à la carte, pour s'aligner sur les pratiques de plus en plus variées d'individus en quête d'une maîtrise de leur espace-temps (Van Eeckhout, 1997 ; Baufils et al., 1999 ; Pény et Wachter, 1999). Nourrie des travaux de chercheurs (Lepetit et Pumain, 1993 ; Obadia, 1997 ; Annales de la recherche urbaine, 1997 ; Annales de la recherche urbaine, 2000), l'administration a ainsi amorcé une réflexion sur le «temps des villes $»^{3}$. Tout ce champ de la littérature décrit la pression qui s'exercerait désormais sur les services publics pour qu'ils s'adaptent à la demande de citadins aisés, devenus plus exigeants. Ces textes contribuent à façonner l'image de consommateurs urbains de services publics et privés ayant la possibilité de « voter avec leurs pieds » en faisant défection lorsque l'offre locale ne satisfait pas et quand une alternative de qualité existe sur le marché privé ${ }^{\text {. }}$ Aucun discours ne se risque toutefois à réduire l'habitant-usager au rang de

\footnotetext{
3 Cf. le rapport Hervé commandité par le ministère de l'Emploi et de la Solidarité (Hervé, 2001).

4 Sur cette application aux services publics de la célèbre grille d'analyse d'Albert O. Hirschmann (Hirschmann, 1972), qui montre comment la défection (exit) peut être moins coûteuse que l'engagement dans une action collective (voice), cf. (Jobert, 1992).
} 
simple client des services publics. Plus conformes aux valeurs républicaines censées inspirer le «service public à la française », les logiques d'usage et de citoyenneté continueraient de prévaloir sur celle de l'utilité (Quin, 1995). Chercheurs et décideurs s'accordent généralement sur ce point : l'habitant des villes françaises n'a pas la connaissance du coût des services qui lui permettrait d'agir rationnellement, dans la logique du public choice (Barthélemy et Leflaive, 1996 ; Rousseau, 1998) ; il se ferait tour à tour usager, client et citoyen sans que l'une de ces figures annule les autres (Chauvière et Gotbout, 1992 ; Joye, 1999).

\subsection{Coordonner par le territoire et l'usager}

Avec la montée en puissance des collectivités locales, la diversification de l'offre de services et leur ajustement à la diversité des demandes posent le problème théorique et pratique de l'intégration de l'action publique. Tel est du moins le problème qu'est amené à se poser l'État, dont les services sont déstabilisés par la remise en cause des méthodes de planification qui avaient connu leur point d'orgue dans les années 1960. Face au poids acquis par les villes, les chaînes de commandement verticales qui présidaient à la fourniture d'équipements et de services publics paraissent inadaptées. L'État d'avant la décentralisation n'était certes pas aussi jacobin que pourrait le laisser penser une partie de la littérature officielle des années 1990. Dès les années 1970, d'importants travaux du Centre de sociologie des organisations montraient que les services extérieurs de l'État étaient loin d'être hermétiques à l'environnement local, qu'ils s'inscrivaient dans des « systèmes de régulation croisée » (Crozier et Thoenig, 1975) ou dans un «système politico-administratif local » (Grémion, 1976) autorisant bien des «arrangements » entre l'État et les élus locaux, surtout les notables ruraux. Après les réformes avortées des collectivités locales dans les années 1960, un courant moderniste, animé par des hauts fonctionnaires de l'État central (issus principalement de la Datar et du ministère de l'Équipement), a promu la méthode contractuelle comme outil d'une responsabilisation pragmatique des élus urbains et ruraux, en même temps que les grilles nationales 
d'équipement étaient progressivement abandonnées ${ }^{5}$. Emblème de la modernisation administrative (Thoenig, 1973), le ministère de l'Équipement va s'affirmer comme le principal interlocuteur des collectivités locales sur les questions d'équipement. Se comportant en «administrations de mission», ses services déconcentrés parviennent à contourner les préfets et contribuent à affaiblir la régulation croisée avec les notables ${ }^{6}$.

Ces premières formules de contractualisation étaient expérimentales et l'esprit de tutelle n'en était guère absent (Nemery, 1981). Mais certains observateurs y ont vu la matrice discrète des partenariats découlant de la décentralisation de 1982-1983 (Gaudin, 1989 ; Lorrain, 1989)7 . Le mouvement paraît désormais irréversible : au modèle rural de l'administration républicaine qui tend à devenir résiduel se serait substitué celui du gouvernement urbain, contractuel et décentralisé (Lorrain, 1991). Dans les années 1990, la science politique s'efforce de conceptualiser ce changement de paradigme. Qu'on l'appelle «système de gouvernement local » (Muller, 1992), « modèle polycentrique » (Gaudin, 1993a) ou «institutionnalisation de l'action collective» (Duran et Thoenig, 1996), il s'agit de décrire un mode de gestion publique fortement différencié selon la nature des enjeux territoriaux et des logiques d'acteurs. Créé en 1992 avec l'appui de différents ministères, le Programme interdisciplinaire de recherches sur les villes (PIR-Villes) du CNRS s'est intéressé aux négociations partenariales dans ce nouveau cadre urbain (ef. Gaudin et Novarina, 1997 ; Verpraet, 1997). Le ministère de l'Équipement se saisira lui aussi de ces thèmes pour se poser cette question; «Qui fait la ville aujourd'hui ?»(Plan urbain, 1997). Ce texte est révélateur d'une orientation forte de la commande publique adressée aux chercheurs pour qu'ils aident l'État à redéfinir ses missions dans un contexte post-

5 Dans le domaine urbain, on fait ici référence aux programmes de modernisation et d'équipement, aux contrats de plan avec les communautés urbaines, à la politique des centres anciens, au programme des villes moyennes, au rôle des agences d'urbanisme, aux procédures Habitat et vie sociale, etc.

${ }^{6}$ Le concept d'administration de mission a été forgée par Edgar Pisani dans les années 1950. Il s'agissait de dépasser le formalisme de l'administration classique pour conférer aux administrations un rôle d'impulsion et de coordination plutôt que de gestion (Pisani, 1956).

7 Le rapport Guichard (Guichard, 1976), qui préfigurait à certains égards les réformes socialistes, préconisait déjà de décentraliser le plus possible ces procédures contractuelles. 
décentralisation. Mais, en quête de nouveaux modèles explicatifs, ce ministère ne semble pas se souvenir qu'il fut le fer de lance de la réforme dans le contexte politiquement conservateur des années $1970^{8}$.

Ne pouvant plus prétendre au monopole dans l'exercice local de ses missions, l'État a mis sa propre réforme au premier plan de ses priorités et entreprend de responsabiliser ses services extérieurs comme de repenser leurs relations à l'environnement local. Il s'inspire pour cela des méthodes du management privé. Après les tentatives d'acculturation aux méthodes de la Rationalisation des choix budgétaire (RCB) dans les années 1968-1974, les années 1980 sont marquées par un renouveau du thème du «management public » qui acquiert ses lettres de noblesse théoriques (Burland et Laufer, 1980 ; Gibert, 1983) et fait l'objet de plans gouvernementaux réguliers, par-delà les alternances politiques ${ }^{9}$. Tous ces programmes poursuivent les mêmes finalités ; rationaliser l'emploi des deniers publics, approfondir la déconcentration administrative, renforcer la coordination interservices et susciter l'adhésion des agents par l'introduction de démarches participatives ${ }^{10}$. Dans un environnement local et international fortement évolutif, les services extérieurs de l'État apparaissaient relativement inertes (Grémion, 1992). Quand est lancé le chantier du Renouveau du service public (circulaire du 23 février 1989), les maires urbains s'étaient déjà affirmés comme de véritables entrepreneurs publics (Lorrain, 1989) et le management public local avait émergé

\footnotetext{
8 Sur le phénomène « d'amnésie » dans l'administration, cf. (Gibert et Thoenig, 1993).

9 Programme Le Garrec sans le gouvernement Fabius, promotion des «Cercles de qualité » sous le gouvernement Chirac, politique de «Renouveau du service public » sous le gouvernement Rocard, «Réforme de l'État» sous le gouvernement Juppé. Pour une mise en perspective historique de la modernisation du service public, cf. (Chaty,1997; Bezès, 2002). Ce dernier montre comment les initiatives politiques des années 1980 seront suivies, dans les années 1990, par une véritable institutionnalisation des programmes de réforme administrative, avec notamment la création en 1995 du Commissariat à la réforme de l'État, remplacé en 1998 par la Délégation interministérielle à la réforme de l'État.

10 De la fin des années 1980 au début des années 2000, les outils mis en place au titre de la modernisation administrative sont pléthoriques ; pôles de compétences, projets de service, centres de responsabilité, contrats de service, programmes pluriannuels de modernisation, formules de contractualisation entre administrations centrales et locales, etc. Appuyé sur des enquêtes de chercheurs (il s'agissait de Luc Rouban, Lionel Chaty et Ehrard Friedberg), le premier bilan officiel de ces démarches était mitigé, tant en ce qui concerne la mobilisation des différents secteurs administratifs que la conduite managériale ou la participation des agents (Fraisse et Serieyx, 1994).
} 
comme thème de recherche à part entière (Cahiers du CNFPT, 1987). La figure du maire comptable de son action et soucieux de rationaliser les services publics locaux n'est pas propre à la France et des recherches s'étaient tournées vers les expériences étrangères, notamment anglo-saxonnes, pour étudier les innovations municipales dans un contexte de rareté financière (Clark et al., 1985) ou les conditions d'évaluation et d'amélioration de l'efficacité des services publics locaux (Allen et Conan, 1987 ; Conan, 1988). C'est aussi dans cette période que l'approche anglo-saxonne des public policies se trouve popularisée en France, invitant à regarder l'État comme un acteur parmi d'autres des politiques publiques (Thoenig, 1985 ; Jobert et Muller, 1987 ; Mény et Thoenig, 1989).

Face au développement spectaculaire des services des collectivités locales et alors que l'État n'a pas a priori de rôle moteur dans la gouvernance urbaine (Le Galès, 1995), la réforme de l'administration territoriale devient un impératif des pouvoirs nationaux. Comme le note Philippe Bezès (Bezès, 2002), confronté à la multiplication désordonnée des arrangements locaux, l'État est à la recherche d'une doctrine intégratrice redéfinissant les responsabilités et missions de chacun dans la gestion territoriale. C'est ainsi que des commissions du Plan prennent pour la première fois l'État lui-même pour objet (Closets, 1989 ; Blanc, 1993 ; Picq, 1994) et questionnent son rôle en regard de la mondialisation et de la décentralisation. Mais contrairement aux années 1970, le ministère de l'Équipement n'est pas en première ligne de cette réflexion, ni des réformes. C'est le ministère de l'Intérieur qui prend l'initiative d'une rationalisation des partenariats locaux à travers une succession de textes législatifs ${ }^{11}$. Ce ministère n'est pas le seul à faire preuve d'activisme: sous la direction de Dominique Voynet, celui de l'Aménagement du territoire et de l'Environnement fait adopter la loi d'orientation pour l'aménagement et le développement durable du territoire

\footnotetext{
${ }^{11}$ La loi sur l'Administration territoriale de la République (6 février 1992) donne une assise législative à la déconcentration, avec l'adoption d'une Charte de la déconcentration, tout en consacrant les collectivités locales comme autorités de plein exercice des politiques publiques ; la loi d'orientation pour l'aménagement et le développement du territoire (loi Pasqua du 4 février 1995) institue un Schéma national d'aménagement et de développement des territoires appelé à préciser les orientations de l'État en matière de développement des services collectifs ; enfin, la loi relative au renforcement et à la simplification de la coopération intercommunale (loi Chevènement du 12 juillet 1999) créé les communautés d'agglomérations.
} 
(LOADDT, 25 juin 1999) qui abandonne le « Schéma national d'aménagement du territoire » institué par la loi Pasqua de 1995, au profit de neuf «Schémas de services collectifs », une démarche qui se veut attentive à la diversité des besoins locaux. De son côté, le ministère de la Fonction publique, de la réforme de l'État et de la décentralisation introduit, à la suite des recommandations du rapport Santel (Santel, 1998), la notion de « Projet territorial de l'État » qui doit préciser, sur la base d'un diagnostic territorial, la place des services déconcentrés dans les politiques locales. En 2000, le ministère de l'Équipement apporte sa pierre à l'édifice avec la loi relative à la Solidarité et au renouvellement urbain (loi Gayssot du 13 décembre 2000) qui instaure notamment les «Schémas de cohérence territoriale », lesquels visent à harmoniser les politiques d'urbanisme, de déplacement et de logement, et peuvent définir les grands projets d'équipement et de services.

La multiplication des dispositifs législatifs, sous-tendue par des réflexions menées en ordre séparé, traduit en partie les rivalités entre ministères. Mais ces réformes sont animées par une intention commune; rationaliser et mettre en cohérence, à la «bonne échelle »12, les interventions territoriales de l'État et des collectivités locales dans le contexte de l'après décentralisation. Le territoire est devenu, pour l'État, le niveau privilégié de la coordination interne à ses services et avec leur environnement ${ }^{13}$. C'est ainsi qu'il faut comprendre la montée en puissance du thème de la «territorialisation» comme mot d'ordre pour la modernisation des services publics de l'État appelés à prendre en compte la diversité des situations locales pour y ajuster leurs prestations et nouer des partenariats ${ }^{14}$. Le processus de territorialisation concerne aujourd'hui la plupart

\footnotetext{
12 Le thème de «l'optimum territorial » a suscité un littérature considérable. Cf. par exemple (Ascher, 1995 ; Ascher, 1998 ; Jouve et Lefèvre, 1999). Dans un contexte urbain qui annonce la « fin du jardin à la française » (Faure, 1999), le «mythe » de l'optimum territorial a souvent été dénoncé par les chercheurs (cf. par exemple (Ortiz, 1994). Pour une revue de la littérature consacrée au sujet cf. (Estèbe et Kirszbaum, 1996)).

13 Sur le concept de coordination dans les organisations décentralisées, publiques ou marchandes, cf. le colloque « Décentralisation et coordination » (Affichard, 1994).

14 À ce sujet, cf. notamment le rapport du Commissariat général du plan sous la direction de Jean-Pierre Delevoye (Delevoye, 1997); cf. également le point de vue de Michèle Cascalès (Cascalès, 1997), universitaire alors chargée de mission au Plan.
} 
des domaines de compétence de l'État et les chercheurs ont été sollicités pour en théoriser les enjeux (cf. par exemple (Charlot, 1994), à propos de l'école ; (Brouant et Jégouzo, 1998) à propos du logement; (Béhar, 1999) à propos des DDE et des politiques d'aménagement; (Commaille, 2000) à propos de la justice). Notons que le processus de territorialisation des services de l'État n'a pas empêché la prolifération de procédures contractuelles que les chercheurs ont regardé tout à la fois comme facteur d'instabilité dans la gestion des territoires et comme outil de coordination (Gaudin, 1996; Marcou et al., 1997). La territorialisation des services accélère le recours aux contrats, l’État restant l'instigateur principal de cette dynamique.

Dans cette recomposition des relations entre les services publics gérés par l'État et leur environnement local, le discours modernisateur fait grand cas des usagers. Mais son ambition est traversée par une ambiguïté de fond: s'agit-il d'améliorer l'efficacité des services en l'instrumentalisant à des fins de coordination, c'est-à-dire d'efficacité, ou bien de modifier l'équilibre des pouvoirs entre l'État et la société civile dans une perspective de démocratisation ? Luc Rouban distingue à ce propos un modèle managérial de la modernisation et un modèle sociétal, vecteur d'une «nouvelle citoyenneté» (Rouban, 1997). L'examen de la littérature consacrée au sujet, à partir des années 1980, suggère en fait un déplacement de l'enjeu. Jusqu'à la fin des années 1970, la mobilisation de la société civile n'était pas étrangère à la recherche d'une plus grande efficacité, mais cet objectif était indissociable d'une démocratisation de l'administration ${ }^{15}$. Il s'agissait, pour le courant modernisateur de l'État, de contourner les forces conservatrices que représentaient le préfet et ses notables (Worms, 1966) en instituant des corps intermédiaires capables d'incarner les «forces vives» de la société et notamment les nouvelles couches moyennes en pleine expansion dans les villes. C'était le thème de «l'administration consultative» (Weber, 1967),

\footnotetext{
15 Pour une riche synthèse de la littérature administrative et savante de cette période, cf. la thèse de Thierry Oblet (Oblet, 1997).
} 
c'est-à-dire de la représentation des intérêts ${ }^{16}$ au sein d'instances techniques (Plan, aménagement du territoire, régionalisme fonctionnel) en charge de l'équipement du pays et, surtout, des territoires urbains. Mais c'est principalement à travers l'animation socioculturelle et les revendications qualitatives sur le cadre de vie, portées par les nouvelles couches urbaines (Gilbert et Saez, 1982), que la participation des usagers a trouvé à s'exprimer durant cette période, qui était aussi celle de la dénonciation par la sociologie critique des «appareils de normalisation» (Cerfi, 1973). La fin des années 1970 signalait également l'arrivée des représentants d'usagers dans les conseils d'administration des entreprises nationales opératrices de réseaux, alors que le mouvement des consommateurs était à son apogée (ef. Jeannot, 1998). Une série de lois venait enfin renforcer le pouvoir des administrés face aux services publics ${ }^{17}$.

À partir des années 1980, les usagers trouvent leur place dans une dynamique de modernisation sans doute moins démocratique que managériale. Dans un premier temps, les entreprises de service public opérant dans le secteur marchand sont les forces motrices de cette dynamique, calquant leurs modèles de management et de relations aux usagers - ici des clients - sur ceux qui ont cours dans les entreprises privées (Strobel, 1993). Alors que se profile la concurrence, l'agglomération des choix individuels a un impact plus certain sur les gestionnaires de ces entreprises que les pressions de groupes organisés; la figure du citoyen associé à la délibération s'éloigne d'autant et la participation de l'usager ne revêt plus une signification politique, sauf à assimiler marché et démocratie comme le font les théories libérales dont l'influence est assez marginale en France (Jeannot, 1998).

Jusqu'à quel point pousser le parallèle avec le marché s'agissant de la modernisation des services publics, notamment non commerciaux ? L'analyse des

\footnotetext{
16 Ceux que l'on désignait comme des «citoyens situés» par opposition aux «citoyens abstraits » de la tradition de 1789. Sur cette conception de la modernisation administrative, cf. (Donzelot, 1986).

17 Loi du 3 janvier 1973 instituant le Médiateur de la République, loi du 6 janvier 1978 sur l'informatique, les fichiers et les libertés, loi du 17 juillet 1978 sur la liberté d'accès aux documents administratifs et loi du 11 juillet 1979 sur la motivation des actes administratif. Pour une vue d'ensemble de l'évolution des textes, cf. (Delaunay, 1993).
} 
interactions entre agents et usagers dans les termes de la « relation de service » a permis d'établir des correspondances entre certaines innovations managériales (amélioration de l'accueil, réorganisation du travail des agents pour la délivrance de réponses plus immédiates, recours aux nouvelles technologies, chartes de qualité, etc.) et l'économie des services appréhendée, elle aussi, dans les termes de la relation de service (Gadrey, 1990 ; Bandt et Gadrey, 1994). Dès 1985, cette thématique a commencé de susciter une intense activité scientifique, épaulée par la commande publique et notamment le ministère de l'Équipement qui trouve dans la relation de service matière à une meilleure compréhension des interactions sociales à l'œuvre dans l'espace urbain (Plan urbain, 1991) ${ }^{18}$. Il ressort de cette littérature foisonnante que la coordination entre agents de base et usagers (appelée aussi «coopération» ou «coproduction») est une dimension cruciale de l'efficacité des services, de nature remédier aux «troubles de la communication » avec l'usager, selon l'expression de Jean-Marc Weller (Weller, 1993). Focalisée sur les micro-interactions, cette approche délaisse sciemment la dimension macrosociale des relations de pouvoir que continuent de privilégier les analyses centrées sur les institutions et les organisations. Cette dernière approche a d'ailleurs pointé les limites d'une modernisation s'accomplissant «par le bas », en montrant la permanence d'une tradition d'assujettissement de l'administré à la puissance publique (Chevallier, 1992 ; Jobert, 1992), d'une culture bureaucratique fondée sur des «normes descendantes »(Gibert et Thoenig, 1993), soulignant aussi le poids des corporatismes et la prégnance des objectifs internes aux

18 Le compte rendu du séminaire sur «La relation de service dans le secteur public», également soutenu par la DRI et la RATP, a permis de familiariser les lecteurs français avec des travaux de la sociologie anglo-saxonne, notamment ceux de Michael Lipsky sur la «Street level bureeaucracy» (Lipsky, 1980). Pour une revue très complète de la littérature consacrée à la relation de service, cf. (Weller, 1998) qui a recensé les nombreux séminaires, appels d'offres et ouvrages collectifs centrés sur cette question et soutenus, pour la plupart, par des financements publics. 
organisations (Chaty, 1997 ; Warin, 1997), autant d'éléments qui maintiendraient l'usager dans une position subalterne ${ }^{19}$.

Pour autant, les travaux sur la relation de service se sont refusés à réduire le processus de modernisation à sa seule dimension gestionnaire, mettant en évidence l'existence de rapports de civilité entre agents et usagers. Dans le prolongement des travaux de Patrick Pharo sur le « civisme ordinaire », le guichet a ainsi pu être regardé comme un lieu du politique $(\text { Pharo, 1985 })^{20}$. Mais sauf à considérer le politique comme la somme des interactions individuelles, on ne peut sans doute pas inférer de ces interactions une avancée dans la démocratisation du service public au sens de l'augmentation du pouvoir collectif des usagers que les anglo-saxons appellent «empowerment». En comparaison de l'engouement suscité par les luttes urbaines des années 1970, le succès scientifique de la relation de service paraît consacrer un certain déclin de l'action collective des usagers. Mais on assiste plutôt à un déplacement de l'enjeu, des travaux récents dessinant l'image d'une «action collective individualisée », à durée limitée et sur des objectifs restreints, inscrite dans la logique des réseaux et reposant sur la valorisation des compétences personnelles plutôt que sur l'adhésion à l'idéologie d'un groupe (Ion, 1997; Neveu, 1999). Emblématique des mutations du militantisme, le cas des associations cherchant à négocier ou s'opposer aux projets d'aménagement, de transports ou d'équipements urbains a fait l'objet, ces dernières années, d'une attention soutenue, notamment par les services de recherche du ministère de l'Équipement ${ }^{21}$, cela dans une période où les textes

19 Le constat d'un déficit presque consubstantiel d'ouverture des services de l'État aux usagers, a été étayé par des études sectorielles sur la police, qualifiée de «boite noire » juxtaposant des «micro-féodalités » (Monjardet, 1996; Monjardet, 1999), ou l'école, dont l'absence de dialogue serait la forme standard de sa relation aux familles (Derouet et Dutercq, 1990 ; Lorcerie, 1998), notamment celles issues des milieux populaires (Dubet, 1997). Cela dans le même temps où ces institutions affirment placer la « demande sociale de sécurité » et « l'élève » au centre de leur action.

20 Telle est du moins l'approche privilégiée par l'un des courants d'analyse de la relation de service distingués par J.-M. Weller (Weller, 1998).

${ }^{21}$ Cf. les recherches de P. Warin (Warin, 1995) et de C. Dourlens (Dourlens, 1996), ainsi que le séminaire de recherche du Plan urbain (Gillio et Ion, 1996) à propos des relations entre les associations et l'administration de l'Équipement ; cf. également le colloque organisé par le Certu (Certu, 2000) et la recherche de C. Atger et al. (Atger et al., 2002) soutenue par le Certu à propos de la participation des usagers à l'élaboration des PDU. 
régissant l'information des citoyens et le débat public se sont succédé à grand rythme ${ }^{22}$. À propos des conflits entre riverains et aménageurs, un nouveau champ de recherches s'est ainsi ouvert avec l'étude du phénomène «Nimby » ${ }^{23}$ (Dear, 1993). Le thème de la participation des habitants semble retrouver une actualité, mais contrairement aux luttes urbaines des années 1970 centrées sur la revendication de nouveaux équipements, le Nimby traduit précisément un refus de voir des équipements dégrader la qualité de vie recherchée dans les espaces périurbains. Les chercheurs proposent deux lectures opposées du phénomène, tantôt interprété comme un «geste de sécession », selon l'expression de MarieChristine Jaillet (Jaillet, 1999 ; cf. aussi Trom, 1999; Lolive, 1999), tantôt appréhendé comme l'irruption de la société civile dans le débat public (TapieGrime, 1997 ; Jobert, 1998 ; Estèbe, 2002)24.

\subsection{L'intérêt général de l'unité à la diversité?}

Quelles sont les conséquences normatives des évolutions décrites (diversification de l'offre, adaptation territoriale et individualisation de la relation aux usagers) ? Les normes du service public seraient désormais écartelées entre unité et diversité (AJDA, 1997 ; DGAFP, 1998a). En suivant la doctrine officielle et de nombreux travaux d'experts, l'héritage des Trente Glorieuses paraît lointain qui faisait de l'État le seul dépositaire et énonciateur d'un intérêt général uniforme en tout point du territoire national. Les travaux de François Dupuy et Jean-Claude Thoenig sur «l'administration en miettes» (Dupuy et Thoenig, 1985) avaient cependant montré comment l'organisation verticale et la

22 De la loi du 12 juillet 1983, dite «loi Bouchardeau », relative à la démocratisation des enquêtes publiques jusqu'aux dispositions de la loi du 27 février 2002 relative à la démocratie de proximité concernant la participation du public à l'élaboration des grands projets, en passant par la circulaire Bianco (15 février 1992) faisant suite à l'affaire du TGV Méditerranée, la «loi Barnier » du 4 février 1995 créant la Commission nationale du débat public et la relance des Plans de déplacements urbains par la loi de l'Air du 30 décembre 1996.

${ }^{23}$ De l'expression américaine « Not In My Backyard».

${ }^{24}$ La place des usagers dans les services publics locaux organisés en réseaux suscite aussi des appréciations divergentes, selon que l'on y voit un pouvoir confisqué par les décideurs politicoéconomiques locaux (Berland et al., 1995) ou l'expression d'une démocratie spontanée «de processus » nourrie des relations quotidiennes entre usagers, opérateurs et élus (Lorrain, 1996). 
réglementation envahissante des services publics renforçaient paradoxalement leur fragmentation, en conférant au local une marge d'autonomie dans l'application des règles. Quelle est donc la spécificité de la période qui s'ouvre avec la décentralisation du point de vue de l'élaboration des normes du service public ? Patrice Duran et J.-C. Thoenig (Duran et Thoenig, 1996) rappellent que la régulation croisée était un ajustement s'opérant en aval de l'action publique, tandis que le nouveau mode gestion territoriale verrait cet ajustement se faire dès l'amont. La puissance publique ouvrirait aujourd'hui des «fenêtres d'opportunité » pour une action collective territoriale, sans en définir a priori la substance. Dans le même sens, Pierre Lascoumes et Jean-Pierre Le Bourhis (Lascoumes et Le Bourhis, 1998) distinguent l'intérêt général «substantiel » et les politiques «procédurales» opérant par la mise en place territoriale d'instruments de connaissance, de délibération et de décision peu finalisés a priori. D'autres chercheurs ont évoqué un «intérêt général local » Gaudin, 1993b) ou «pluraliste » (Nemery et Wachter, 1994), voire une «territorialisation du droit» (Madiot, 1995). Ces thèses n'ont pas tardé à inspirer des rapports officiels (cf. en particulier le rapport Delevoye, déjà mentionné, et celui que le Conseil d'État a consacré à « l'intérêt général » en 1999).

Un second champ de réflexions sur la production des normes du service public s'est ouvert avec l'ouverture à la concurrence des entreprises nationales opératrices de réseaux et le débat sur les délégations au secteur privé de la gestion des services publics locaux - ce dernier débat ayant pris de l'ampleur avec la médiatisation des «affaires ». Dans les deux cas, pouvoirs publics et chercheurs, se sont intéressés à l'invention de nouvelles « régulations », mais le concept de régulation n'y revêt pas le même sens. Dans le cas des entreprises autrefois monopolistiques, la régulation est à comprendre au sens anglo-saxon, comme la réglementation et le contrôle des opérateurs de services. Ici, les réflexions publiques se sont ouvertes aux exemples étrangers pour chercher les voies d'une conciliation entre services intérêt général et efficacité économique (Stoffaës, 1995 ; Stoffaës, 1997 ; Attias, 1997 ; Henry, 1999 ; Ministère de l'Équipement, 1999). À propos des services publics locaux, D. Lorrain (Lorrain, 1990) a 
souligné à quel point ce «second modèle» était éloigné de la conception régalienne d'un État situé au-dessus de la société et édictant des normes. Ce second modèle se caractériserait par une plasticité des formes juridiques où prédominent les formules contractuelles. Ici, l'enjeu de la régulation réside moins dans l'édiction de normes juridiques (hormis la question de la transparence des marchés soumises, elle aussi, au droit communautaire) que dans la nature du pilotage de l'action publique locale. Après avoir longtemps négligé cette dernière question, la réflexion publique et scientifique se tourne aujourd'hui vers les conditions d'une maîtrise, par les décideurs publics locaux, des services confiés au opérateurs privés (Annales des Mines, 1994 ; Drouet et al., 1996 ; Martinand, 2001). La structure de choix des collectivités est désormais plus ouverte, mais certains travaux ont pointé le danger d'une perte d'autonomie des décideurs publics (Davezies et Prud'homme, 1993 ; Plan urbain, 1997 ; Coing, 1998). D'autres ont souligné, au contraire, les capacités d'autorégulation du système (Lorrain, 1993), et ont vu dans l'extension du marché une opportunité offerte aux collectivités de conforter leurs positions (Offner, 1995 ; Cahiers du génie urbain, 1996).

Une troisième manière, très différente, d'envisager la formation d'un intérêt général local renvoie au travail des agents. Dans leur ouvrage déjà mentionné, F. Dupuy et J.-C. Thoenig évoquaient déjà une «pyramide inversée » au sein de laquelle les agents disposeraient d'une certaine liberté dans l'application de la loi. Mais la reconnaissance d'une production normative des agents reste l'un des acquis théoriques importants du courant d'étude de la relation de services. Qu'elles s'inspirent de l'école conventionnaliste (Boltanski, 1992) ou de l'interactionnisme goffmanien (Jeannot et Joseph, 1995), ces approches ont mis en évidence la pluralité des normes susceptibles d'être invoquées par les agents dans leur travail au contact des usagers. De nombreuses recherches ont été commanditées par les pouvoirs publics sur cette problématique. À propos de l'école, Jean-Louis Derouet (Derouet, 1992) a montré comment, en l'absence d'un référent national incontestable, les enseignants sont contraints de mettre en œuvre un important travail d'interprétation de la norme. Dans le cas des DDE, 
Philippe Corcuff et Claudette Lafaye (Corcuff et Lafaye 1993) ont relaté la manière dont les agents sont amenés à définir eux-mêmes, au cas par cas, la conception du service public et de l'intérêt général. Une recherche de François Eymard-Duvernay et Emmanuel Marchal (Eymard-Duvernay et Marchal, 1996) a mis en évidence, à propos d'une innovation dans un office HLM, deux formes de coordination coexistant simultanément dans l'organisation, selon que les agents s'ajustent aux locataires en invoquant des règles générales définies à l'avance ou se réfèrent à la négociation en direct avec les habitants. La relation de service contribuerait ainsi à pluraliser et localiser les normes du service public, le territoire où se joue la relation avec l'usager se trouvant investi d'une fonction de création de normes, au lieu de n'être qu'un point d'application passif de celles-ci.

\section{Les services publics face à l'éclatement de la ville}

\subsection{Ségrégation spatiale et inégalités devant le service public}

L'idée d'une distance sociale aux services publics, en particulier à l'école et aux équipements culturels, fut l'un des thèmes favoris de la sociologie critique des années 1960 et 1970, notamment celle de Pierre Bourdieu. Une recherche conduite par le CSU (Culture et sociétés urbaines) à partir de 1975, avec le soutien du Commissariat général du plan et du ministère de l'Équipement, s'est efforcée, pour la première fois, de combiner une analyse sociale et spatiale de l'usage des équipements et services par le croisement systématique d'indicateurs sur les classes sociales et la présence des équipements collectifs dans les communes de la région parisienne. L'étude concluait à l'existence d'une ségrégation qui réserve aux communes aisées l'usage des meilleurs équipements et repousse les classes dominées vers les zones de banlieue les moins bien dotées (Préteceille et al., 1986). Cette recherche ne spécifiait pas, cependant, la situation des territoires infracommunaux, en particulier celle des quartiers de grands ensembles en proie à une spécialisation sociale et ethnique croissante, et qui 
allaient devenir la figure emblématique de l'inégalité urbaine devant les services publics. La question avait commencé d'être officiellement posée par le groupe interministériel Habitat et vie sociale (1977), dont l'une des missions était de satisfaire les besoins en équipements et services collectifs dans la cinquantaine de quartiers retenus au titre de ce programme expérimental. Par la suite, les rapports fondateurs de la politique de la ville (Schwartz, 1981; Bonnemaison, 1982 ; Dubedout, 1983), puis les premiers bilans des opérations de Développement social des quartiers (Pesce, 1984 ; Lévy, 1988), ont formulé des diagnostics convergents sur les difficultés quotidiennes subies par les habitants de ces quartiers dans différents domaines (emploi, sécurité, école, santé, action sociale, transports, etc.) et sur l'inadaptation quantitative et qualitative des équipements et services, qu'ils soient gérés par l'État ou les collectivités locales. Mais les services publics de l'État n'étaient toujours pas constitués en objet propre de l'analyse, ni des réformes préconisées par ces commissions composées de techniciens de l'État et d'élus. Seuls les services des villes avaient fait l'objet d'une réelle attention (CNFPT et CNDSQ, 1987). De son côté, la doctrine du ministère de l'Équipement restait attachée à ses objets traditionnels (logement, aménagement, transports), envisagés sur un plan essentiellement technique.

Il faut attendre la création d'un ministère de la Ville, en 1990, pour que les services publics - de l'État comme des collectivités - soient traités, au plan national, comme une question à la fois globale et décisive pour l'avenir de ces quartiers (c'est ainsi que l'un des départements de la Délégation interministérielle à la ville prend en charge, en 1991, «les services au public »). À la suite du séminaire gouvernemental du 7 décembre 1990, le ministère de la ville avait confié à trois inspecteurs de l'administration centrale une mission d'étude sur la présence des services publics dans les quartiers. Leur rapport dressait un état des lieux sévère (Langlais et al., 1991). Négociés en 1993, les contrats de ville devaient faire en conséquence de l'adaptation quantitative et qualitative des services leur thème d'action numéro un (DIV, 1993). Le gouvernement de droite revenu au pouvoir cherchera à systématiser cette approche, en dressant par exemple un état des lieux exhaustif de la présence des services publics dans les 
quartiers, avec l'espoir de définir une norme d'équipement. Mais la base de données centrale ne sera jamais alimentée. Les services publics ressortent alors comme une dimension essentielle de la stratégie de réduction des « handicaps territoriaux » dont la loi Pasqua sur l'aménagement du territoire (1995) a fait son mot d'ordre et que le Pacte de relance pour la ville, adopté sous le gouvernement Juppé (1996), se chargera de mettre en application dans les banlieues déshéritées $^{25}$. De retour aux affaires en 1997, la gauche s'est démarquée de la méthode du zonage pour revenir à la logique contractuelle. Mais elle a confirmé la ligne «républicaine» faisant du déficit des services publics dans certains territoires un critère essentiel de l'inégalité entre citoyens. S'appuyant sur diverses études sectorielles produites par les administrations, la commission Sueur (Sueur, 1998) a ainsi méthodiquement recensé les inégalités d'implantation des services publics gérés par l'État (police, justice, éducation, poste, emploi...). Les perceptions locales du problème ne sont toutefois pas forcément les mêmes. Ainsi a-t-il été demandé aux préfets d'établir un diagnostic, duquel il ressort que la sous-administration des quartiers est un cas de figure assez minoritaire. Les déficits seraient désormais moins quantitatifs que qualitatifs (accueil, écoute, horaires, repérage...) (Bengaouer et Pestre-Mazières, 1999). Alors même que les services publics sont officiellement présentés comme la pierre angulaire d'un modèle républicain à préserver ou rétablir dans ces territoires «sensibles », on notera que bien peu de commandes ont été adressées aux chercheurs pour acquérir quelques certitudes sur 1'offre réelle de ces services dans les quartiers pauvres ${ }^{26}$.

L'une des questions centrales de la recherche, dans les années 1990, porte sur la contribution des services publics à la ségrégation des usagers habitant ces territoires et, plus précisément, sur l'impact ségrégatif des logiques marchandes et $\mathrm{du}$ « souci de la performance » qui accompagnent la diversification de l'offre de services (cf. supra, première partie). Depuis que la société est confrontée au

\footnotetext{
25 Le Pacte de relance faisait aussi le pari du développement économique avec la création des Zones franches urbaines.

26 Une des rares études d'envergure sur le sujet, mais plus qualitative que quantitative, est celle d'Annie Maguer et Jean-Marc Berthet (Maguer et Berthet, 1997) initiée par le ministère de la Fonction publique.
} 
chômage de masse et à la paupérisation d'une frange de la population, les services publics sont confrontés à un phénomène parfois qualifié de «débordement du social » (Informations sociales, 1999), dont l'intensité est maximale dans les quartiers d'habitat social. Les institutions réagiraient à cette nouvelle situation en combinant deux options : différencier toujours davantage leurs prestations pour continuer à servir des catégories d'usagers de moins en moins homogènes (Kaysergruber et Strobel, 1996) et/ou organiser un partage des tâches avec le secteur marchand voué à servir les seuls ménages solvables (Landrieu, 1998 ; Warin, 1999). Transposées à l'urbain, ces analyses suggèrent un rôle actif des services publics dans la ségrégation des quartiers pauvres auxquels serait réservée une offre de second choix, le marché ayant pour fonction de remplir des fonctions que ces habitants captifs ne peuvent s'offrir.

Cette thèse a été développée principalement à propos du logement et de l'école, deux secteurs très sensibles aux effets de la «démocratisation », c'est-àdire à la prise en charge de publics nouveaux, notamment d'origine immigrée. La réforme du logement de 1977 entendait «déspécialiser» l'offre destinée aux populations modestes et faciliter l'accès à la propriété. Son bilan montre pourtant une tendance à la fragmentation accrue de l'offre de logements sociaux, liée à la multiplication de «produits» ajustés à chaque type d'occupants (PLA, PLI, PLAI, habitat adapté, etc.). Or, les produits réservés aux ménages les plus modestes ont été concentrés dans les mêmes quartiers, contribuant à la ségrégation spatiale de ces ménages (Tanter et Toubon, 1995). Dans le même temps, la réforme de 1977 a eu pour effet de contracter la production aidée au profit du marché privé, lequel a fini par constituer le segment dominant de l'offre, notamment dans le tissu périurbain. L'accession à la propriété devenant moins sociale, plus dépendante des banques et des opportunités fiscales, les ménages les moins solvables sont de plus en plus captifs des segments les moins attractifs de l'offre sociale. Des chercheurs ont ainsi dénoncé la création d'un «duopole» (Coloos, 1995) ou « l'autonomisation » du volet social (Ballain et Béhar, 1998), fondée sur un «principe d'exclusion» mutuel avec le marché (Lapierre, 2000), 
une contradiction reconnue par certains responsables du ministère de l’Équipement (Landrieu, 1997).

Les chercheurs ont abouti à des constats similaires concernant l'école. Éclatée entre quartiers riches et pauvres, une offre scolaire de plus en plus différenciée augmenterait les choix - au sein du secteur public; entre écoles publiques et privées - de ceux qui habitent les premiers et contribuerait à la captivité des autres au sein des établissements publics les moins cotés (Husson et Klein, 1991 ; Champion et Tabard, 1996). Parce qu'elles sont insuffisamment compensées par l'État, les inégalités de richesses entre communes aggraveraient cette tendance, depuis que les municipalités sont devenues des acteurs importants des politiques scolaires (Thin et al., 1992). Cette offre scolaire différenciée contribuerait, dans une certaine mesure, à creuser les divisions sociales de l'espace au détriment surtout des quartiers à forte population immigrée (Van Zanten et al., 1991 ; Rhein et al., 1996 ; sur l'ethnicisation des politiques scolaires (Payet, 1995)). Comme pour le logement, la structure éclatée de l'offre scolaire ne serait pas le simple reflet de la ségrégation urbaine, mais contribuerait aussi à l'organiser. Cette idée est désormais admise dans des rapports officiels du ministère de l'Éducation nationale (Toulemonde, 1998) ou adressés à lui (Dubet, 1999)27.

Des résultats de ces recherches sectorielles, les responsables nationaux ont parfois tendance à s'emparer pour généraliser l'idée «d'un service public » contribuant à produire l'exclusion dans les villes en raison de sa soumission aux impératifs de performance et de rentabilité (Brévan-Picard, 2000). Cette généralisation va jusqu'à englober les entreprises nationales opératrices de réseaux, au risque de négliger leurs propriétés spécifiques ${ }^{28}$. Posé à partir de la

\footnotetext{
${ }^{27} \mathrm{Cf}$. également le programme de recherches « mixité urbaine et ségrégation à l'école » engagé, en 2000, par le ministère de l'Éducation nationale, le Puca, le FAS et la DIV.

28 Cf. les conclusions de l'ouvrage sur la «ville éclatée », par Josée Landrieu, chargée de mission au ministère de l'Équipement, qui a coordonné le séminaire organisé sur ce thème par le ministère (Landrieu, 1998).
} 
question du « service universel $»^{29}$, le débat européen sur le « service public à deux vitesses » ne peut être transposé sans précaution aux quartiers urbains défavorisés. La remise en cause des péréquations tarifaires peut certes renforcer les inégalités d'accès, mais le risque de «débranchement » paraît davantage lié aux ressources financières des ménage ${ }^{30}$ qu'à leur lieu de résidence, étant donnée la couverture territoriale des réseaux. Les chercheurs ne se risquent généralement pas à établir une corrélation entre l'ouverture à la concurrence des entreprises nationales et les processus de ségrégation des quartiers d'habitat social ${ }^{31}$. La dimension proprement spatiale de cette question renvoie en fait aux équilibres entre territoires ruraux et urbains. Face au calendrier imposé par Bruxelles, les responsables des entreprises concernées sont demandeurs d'un débat public sur le maintien de péréquations géographiques qui continuent d'avantager le monde rural (Stoffaës, 1995 ; Flux, 1995).

En dehors du cas particulier des entreprises opératrices de réseaux, cette dernière question est souvent abordée sous l'angle du redéploiement des effectifs vers les zones urbaines, la situation des communes pauvres de banlieue étant souvent invoquée comme une justification à ces redéploiements (Verdeil, 1998 ; Bodiguel, 1999). Il s'agit là d'un enjeu politique où des nuances sont sensibles entre la droite et la gauche : le thème du maintien des services publics en milieu rural était au cœur de la loi Pasqua sur l'aménagement du territoire (1995), tandis que l'administration Voynet est apparue d'inspiration plus urbaine, faisant de la

\footnotetext{
${ }^{29}$ La notion de «service universel » a été introduite par Bruxelles pour compenser les effets sociaux de l'application du prix du marché dans les services en réseaux. Le service universel est défini comme «le service de base offert à tous, dans l'ensemble de la Communauté, à des conditions tarifaires abordables et avec un niveau de qualité standard $»$ (Livre vert sur les services postaux, 1992). Les entreprises de réseaux françaises ont commencé d'intégrer cette logique avec la signature, en 1996, d'une charte «solidarité » dans les domaines de la fourniture d'eau et d'énergie. Puis la loi sur les télécommunications a inscrit le principe d'un abonnement social et l'on retrouve semblable préoccupation à La Poste avec la notion «d'accès minimal au secteur bancaire ».

${ }^{30}$ Ces risques d'insolvabilité ont été soulignés, sous la précédente législature, par des rapports de parlementaires socialistes concernant, par exemple, l'électricité (Bataille, 1999) ou le gaz (Bricq, 1999).

31 À l'exception du cas de la Poste dont les établissements, contrairement à EDF, GDF ou au téléphone, jouent un rôle de premier plan dans le lien quotidien avec les habitants Sur la Poste et les quartiers de la politique de la ville, cf. (Rosenberg, 1997). Pour une analyse plus générale des «prestations de cohésion sociale » de cette entreprise, cf. Gadrey, 1997.
} 
lutte contre les effets de la périurbanisation l'un des axes forts sa politique d'aménagement. Un rapport sur la «ville durable » commandité par ce ministère estimait ainsi que la demande d'équipements et d'infrastructures des périurbains, surtout aisés, absorbait une part croissante d'une richesse collective qu'il serait plus juste de consacrer aux quartiers urbains dégradés (Sauvez, 2001).

2.2. Les «quartiers sensibles» ou les apories de la coordination dans la ville éclatée

Les premières années de la politique des quartiers ont été marquées par une floraison de dispositifs transversaux (opérations de DSQ, conseils de prévention de la délinquance, missions locales, Banlieues 89...) et d'actions éparses conduites par les administrations. La création de la DIV (Délégation interministérielle à la ville), en 1988, fut la première tentative significative de coordination de ces interventions dispersées qui constituaient autant de «guichets» pour les municipalités (Donzelot et Estèbe, 1992). Au début des années 1990 , le thème du « retour de l'État » sur la scène des quartiers faisait son entrée en force. Il s'agissait de rééquilibrer les relations avec les collectivités locales et d'assurer une mobilisation des services de l'État à hauteur de l'enjeu (à ce sujet, cf. le rapport Delarue, 1991). Au plan national, la création d'un ministère de la Ville (1990) devait assurer une fonction de coordination centrale et, localement, l'administration préfectorale devait être le lieu de la coordination interministérielle grâce à l'engagement du préfet, secondé par un «sous-préfet à la ville » auquel était transféré une enveloppe financière (le Fonds d'intervention pour la ville) ponctionnée sur les budgets de plusieurs ministères. L'institution des délégués de l'État (1996), interlocuteurs uniques de l'État dans les quartiers, parachevait cette stratégie de coordination interministérielle. Vis-à-vis des collectivités locales, la procédure «Contrat de ville» devait fédérer l'ensemble contractualisations liées à la politique de la ville et clarifier les relations de l'État avec ses partenaires au niveau intercommunal (Geindre, 1993). 
Sur tous ces plans, les bilans officiels comme les évaluations confiées à des chercheurs ou des cabinets de consultants durant le XI Plan (1994-1999) ont été décevants ${ }^{32}$. Très vite, Dominique Damamme et Bruno Jobert (Damamme et Jobert, 1995) ont souligné les écueils de la coordination interministérielle nationale, la DIV se confrontant à la résistance de puissantes administrations centrales (Équipement, Affaires sociales, Éducation nationale). Localement, la mobilisation des services déconcentrés de l'État s'est avérée fort inégale d'un département à l'autre. Ni le préfet, ni le sous-préfet à la ville ne se sont affirmés comme les orchestrateurs d'une véritable interministérialité (Grémion et Mouhanna, 1995 ; Bachelet et Rangeon, 1996). À la fin du X Plan (1989-1993), les évaluateurs nationaux de la politique de la ville avaient cru voir dans la formule du Contrat de ville, alors expérimentée dans treize sites intercommunaux, l'avènement d'une scène locale permettant un questionnement mutuel de l'État et des collectivités sur l'action des services publics en direction des quartiers pauvres (Donzelot et Estèbe, 1994). Quelques années plus tard, les mêmes chercheurs « réévaluent la politique de la ville », reconnaissant que l'État n'a pu s'affirmer comme le garant des solidarités locales (Donzelot et Estèbe, 1998). L'émergence d'un «État animateur » n'a jamais été qu'embryonnaire en raison d'une intercommunalité de façade - on ne qu'être frappé de ce point de vue par la similitude des constats établis par les rapport Geindre en 1993 et Sueur en 1998 - et de la fragmentation des acteurs comme des dispositifs ${ }^{33}$. Largement municipalisée, cette politique s'est cantonnée en grande partie au rôle de guichet pour le milieu associatif ${ }^{34}$.

Nonobstant d'inévitables rivalités, le processus de territorialisation des services publics amène les institutions à coopérer entre elles. Mais on s'en tient largement à un partenariat entre spécialistes et les quartiers relégués seraient la

\footnotetext{
32 Pour une synthèse de ces évaluations, cf. (Kirszbaum, 1998).

33 Sur l'illisibilité de la politique de la ville, déjà dénoncée par le rapport Delarue, cf. la critique de la Cour des comptes dans son rapport consacré à cette politique en 2002 (Cour des comptes, 2002).

34 Pour une vision peu critique du rôle des associations dans la politique de la ville, cf. le rapport Sandrier (Sandrier, 2001).
} 
«part maudite de la gouvernance urbaine », selon l'expression d'Anne Querrien (Querrien, 1999). Sur ces territoires, ce n'est pas la coopération mais le conflit qui paraît régir la relation des services publics aux populations marginalisées. S'érigeraient entre ces deux mondes des frontières symboliques sur fond d'ethnicisation des rapports sociaux (Moulin, 1997). Il s'agit là d'une situation que l'approche de la modernisation par la relation de service n'a pas toujours bien prise en compte, les institutions étant tentées d'attribuer l'échec de cette relation au déficit de compétences des usagers, lié à leur trop grande distance sociale et culturelle (Macé, 1997a). Les recherches conduites sur cette question, avec des financements publics, ont souligné la « spécificité » du travail des agents en poste dans ces quartiers (Bautier et al., 1995 ; Maguer et Berthet, 1997). Ils y vivraient leur nomination comme un déclassement professionnel, la spécificité de leur travail étant insuffisamment reconnue par les règles de gestion du personnel. Les travaux de Suzanne Rosenberg (Rosenberg, 1995), qui avait joué un rôle éminent dans l'élaboration du rapport Picard (Picard, 1991) consacré à cette question, ont souligné également l'absence de valorisation par l'encadrement du savoir des agents terrain.

L'année 1991 fut marquée par une avalanche de rapports officiels qui multipliaient les propositions visant à atténuer la souffrance des agents dans ces quartiers (outre le rapport Picard, il s'agissait des rapports Langlais et al., Pêcheur et Delarue, déjà cités, ainsi que du rapport Carrère (Carrère, 1991)). Ces textes formulaient les mêmes constats négatifs concernant les conditions de nomination, de cadres d'emploi ou de définition des fonctions de ces agents. Faisant suite aux recommandations du rapport Pêcheur (Pêcheur, 1991), divers avantages (valorisation de carrière, nouvelle bonification indiciaire, priorité de mutation, développement de formations spécifiques) ont été finalement accordés aux fonctionnaires de l'État. Mais faute de réelle prise en compte de leur expérience dans la définition des orientations de la politique de la ville, ils éprouveraient peu de gratitude envers celle-ci pour les mesures préférentielles qu'elle a suscitées (CRIF et PRIF, 1999). 
Frustrant dans les rapports avec la hiérarchie, le travail des agents de terrain ne le serait pas moins avec les habitants. Cette question a fait toutefois l'objet de deux lectures contrastées. Les enquêtes coordonnées par P. Bourdieu sur «la misère du monde »(Bourdieu, 1993) ${ }^{35}$ suggéraient une similitude de condition entre agents et usagers dans ces quartiers, les deux parties étant pareillement victimes de la conversion de la «noblesse d'État» au néolibéralisme (dans le même sens, cf. les travaux déjà cités de Suzanne Rosenberg, Annie Maguer et Jean-Marc Berthet). À l'inverse, les chercheurs de l'école tourainienne, affiliés au Cadis (Centre d'analyse et d'interventions sociologiques), ont avancé l'hypothèse d'un «colonialisme interne », repérable dans la surreprésentation, au sein des services publics, de fonctionnaires issus des classes moyennes venues du dehors et engagés dans une entreprise « civilisatrice » (Dubet et Martuccelli, 1998). Les violences urbaines les visent directement, car ces agents sont culturellement coupés du «contre-monde » des « cités » (Lapeyronnie, 1999). Ces chercheurs n'interprètent pas la crise des services publics comme la conséquence d'une pénalisation de l'État social, à la manière des épigones de P. Bourdieu (ARSS, 1998), mais comme le signe d'une perte de légitimité des institutions incarnant l'idée républicaine. Les discours institutionnels pétris de références aux valeurs de la République apparaîtraient trop décalés en regard des réalités quotidiennes des habitants pour ne pas être ressentis comme une duperie, puisque ces services ne leur donnent pas accès aux ressources sociales et culturelles nécessaires à la construction de parcours personnels (Wieviorka, 1992 ; Wieviorka, 1999). Dans cette perspective, la violence est un phénomène co-construit avec des institutions qui excluent, et dont les agents cultiveraient des comportements hostiles aux habitants. Répondant à des commandes publiques, des travaux concernant la police (Wieviorka, 1991), l'école (Dubet, 1994) ou les transports publics (Macé,

\footnotetext{
${ }^{35} \mathrm{Ce}$ travail avait bénéficié de financements de la Caisse des dépôts et consignations.
} 
1997b) sont venus soutenir cette thèse ${ }^{36}$. L'existence d'une «violence institutionnelle», sur fond de représentation insuffisante des populations d'origine immigrée dans les services publics, a bénéficié d'une tribune dans certains textes officiels (DIV, 1999 ; Brévan-Picard, 2000) ${ }^{37}$.

\subsection{Le spectre du renoncement à la norme d'universalité}

Avec l'exacerbation des disparités sociospatiales, la norme d'égalité de tous devant et par le service public court le risque d'apparaître comme une pétition de principe. La critique de cette conception formelle de l'égalité a été élaborée dans le creuset de la « deuxième gauche », à partir de la fin des années 1980, à travers les essais « grand public » d'Alain Minc qui fustigeaient la « machine égalitaire » (Minc, 1987) et vulgarisaient des concepts anglo-saxons tels que la discrimination positive, ou dans les cercles d'experts liés au Plan (Affichard et Foucauld, 1992) qui se référaient aux philosophies américaines de la justice sociale, tout particulièrement à celle de John Rawls. C'est dans ce contexte intellectuel que la circulaire Rocard sur le renouveau du service public (1989) a placé l'équité au cœur de la démarche de modernisation pour signifier la nécessité d'un compromis entre les exigences d'efficacité et de justice sociale ${ }^{38}$. Peu à peu, les concepts d'équité et de discrimination positive vont envahir les débats français sur la justice sociale, la discrimination positive prolongeant l'équité en lui assignant une finalité explicite de réduction des inégalités (Affichard, 1995). Cette notion avait été officiellement mise en avant par le rapport Schwartz (Schwartz, 1981) sur l'insertion des jeunes. L'idée n'était pas entièrement inconnue; elle avait été

\footnotetext{
${ }^{36}$ Le courant tourainien est loin d'être le seul à s'être penché sur ces questions et notamment sur l'école qui, placée au cœur des contradictions du modèle républicain, a suscité la littérature la plus dense sur les relations avec le public des quartiers en difficulté, souvent en réponse à des commandes de l'Éducation nationale ou de l'Institut des hautes études sur la sécurité intérieure (Charlot et Emin, 1997 ; Debarbieux, 1996 ; Pain et al., 1997 ; Dossiers d'éducation \& formations, 1997). La dimension proprement ethnique de ces tensions a fait l'objet de plusieurs numéros de la revue Ville-école-intégration (VEI, 2000 ; VEI, 2002).

37 Cf. aussi le rapport de Christian Mouhanna, commandité par la DIV, sur « les services publics et la question jeune » (Mouhanna, 1999).

${ }^{38}$ Michel Rocard avait été la figure de proue de la deuxième gauche.
} 
empruntée, par la Sgen-CFDT, à l'expérience britannique des zones d'éducation prioritaire. L'instauration d'un tel programme en France, à partir de 1981, avait été comprise comme l'introduction d'une logique de discrimination positive, sans toutefois que l'expression figure officiellement dans les textes ${ }^{39}$.

Il faut attendre le début des années 1990 pour que ce concept émerge véritablement dans le discours des pouvoirs publics, à propos notamment de la politique de la ville. La référence à la discrimination positive revenait alors à formuler une exigence en direction des services publics pour qu'ils se renforcent dans les quartiers, en quantité comme en qualité (en ce sens, cf. les rapports Picard et Delarue). Les contrats de ville devaient fournir un cadre, celui de la «géographie prioritaire» de la politique de la ville, où se formaliserait l'engagement des services publics à accomplir cet effort prioritaire. Leur bilan a été généralement jugé décevant sous cet aspect, notamment parce que les crédits «spécifiques » de la politique de la ville se seraient substitués aux financements de «droit commun» des administrations. Mais peu d'études ont permis de mesurer avec précision la réalité la discrimination positive territoriale. Le seul travail d'évaluation de quelque envergure (plus qualitatif que quantitatif, il est vrai) a été conduit dans la région Île-de-France au cours du XI ${ }^{\mathrm{e}}$ Plan. Il montre que la géographie prioritaire de la politique de la ville n'a pas été plébiscitée par les services publics, ni comme espace d'analyse, ni comme espace pour une action préférentielle (Crif et Prif, 1999).

Un autre usage officiel a été fait de la discrimination positive territoriale, entendue cette fois comme un ensemble de mesures dérogatoires réservées à des «zones », selon le degré de gravité de leurs handicaps. Cette approche était celle de la loi Pasqua sur l'aménagement du territoire qui posait le principe de «politiques renforcées et différenciées » dans les «zones caractérisées par des handicaps ». Le Pacte de relance pour la ville (1996) a été chargé de traduire ces principes dans des zones cartographiées par la DIV et l'Insee. À la différence des contrats de ville, le principe de la dérogation était inscrit dans le droit et ne

\footnotetext{
${ }^{39}$ Pour cette mise en perspective historique des ZEP, cf. (Bouveau, 1997),
} 
relevait plus d'un simple message volontariste à l'intention des administrations. Dans sa réflexion sur le principe d'égalité, le Conseil d’État (1996) a validé la méthode : «L'intégration civique, culturelle et économique des personnes vivant dans les zones urbaines en difficulté relève d'une cause d'intérêt général et justifie des mesures spécifiques que notre droit accepte désormais. » Prenant soin de souligner la différence avec les politiques d'affirmative action, le Conseil d'État évoquait le traitement différencié du territoire comme l'une des formes les plus actuelles d'une discrimination positive «à la française ». Cette démarche revêt ainsi une signification implicite, celle de surmonter le hiatus né de l'impossible spécification de bénéficiaires «ethniques» dans le modèle universaliste français alors même que ces populations regroupées dans certains quartiers subissent une situation inégalitaire.

Associée à une philosophie politique «de droite » à partir de l'alternance de $1993^{40}$, et compte tenu de son cousinage américain, la discrimination positive est passée à la trappe du discours officiel sous le gouvernement Jospin. Rapidement, une série de rapports officiels a cherché la caution d'experts ${ }^{41}$ pour porter le fer contre cette conception de l'action publique dont divers inconvénients ont été pointés ; catégorisation arbitraire aboutissant à une inflation des «ayants droit » territoriaux, conception réparatrice d'une action publique focalisée sur les symptômes au lieu de traiter leurs causes situées dans les évolutions structurelles de la ville, effets de frontière attisant le ressentiment des populations limitrophes, stigmatisation préjudiciable aux bénéficiaires, etc. (Delevoye, 1997 ; CNV, 1998 ; Sueur, 1998 ; Bravo, 1999).

À cette critique de la discrimination positive territoriale comme méthode d'allocation des ressources publiques, des chercheurs ont ajouté celle du «traitement différencié » des usagers comme nouvelle figure normative de l'action des services publics. Le processus de modernisation entendait confier aux

\footnotetext{
${ }^{40} \mathrm{Cf}$. les violentes polémiques qui ont suivi la publication du rapport Minc sur « la France de l'an $2000 »$ pour le Commissariat général du plan (Minc, 1994). Ce rapport qui devait servir de plateforme au candidat Balladur semblait opposer l'équité à l'aspiration même à l'égalité.

${ }^{41}$ De ce point de vue, les experts les plus influents ont été Daniel Béhar et Philippe Estèbe. Cf. leur commentaire critique du Pacte de relance pour la ville dans la revue Esprit (Béhar et Estèbe, 1996).
} 
agents de base une plus grande autonomie dans la conception de l'offre de services, mais elle les aurait laissés seuls pour résoudre les contradictions nées de la multiplication de registres de référence incompatibles (rentabilité contre insertion sociale, égalité contre efficacité, qualité contre quantité) (Rouban, 1994). Des recherches insistent sur les conflits de légitimité d'agents soumis aux impératifs de rentabilité de leur organisation, et ne sachant plus s'ils doivent appliquer une norme marchande ou de solidarité, par exemple dans les CAF (Gautrat, 1996) ou à la Poste (Rosenberg, 2000). Il en résulterait un brouillage du sens de la mission de service public, glissant d'une conception civique et unitaire vers une conception marchande porteuse d'une segmentation du public en diverses catégories de clients (Corcuff et Lafaye, 1996).

\section{Le souci du rapprochement}

\subsection{Les services publics entre proximité et accessibilité}

Le thème de la proximité des services publics dans les quartiers en difficulté, comme modalité d'une territorialisation de l'action publique, est pratiquement aussi ancien que la politique de la ville elle-même. Déjà, le rapport Dubedout (Dubedout, 1983), fondateur de la démarche de Développement social des quartiers, posait le principe; «rapprocher les services du terrain et des populations », afin de résorber "l'éloignement spatial conjugué à l'éloignement social ». Par la suite, cette orientation a été systématisée, surtout après les assises de Bron, organisées par Banlieues 89 en décembre 1990, au cours desquelles François Mitterrand évoqua la «remise à niveau» du service public dans les «quartiers dégradés ». Le premier des ministres de la Ville, Michel Delebarre, en a fait une priorité majeure de son action, ce dont témoignent les quatre rapports commandés sur le sujet en 1991 (cf. supra). Cette orientation s'est concrétisée dans une stratégie de rapprochement physique conçue pour restaurer les conditions d'un traitement plus égalitaire des quartiers prioritaires (DIV, 1995). 
Mais il s'est agi aussi d'une exigence qualitative, celle d'un ajustement du service rendu à l'environnement particulier que sont ces quartiers, afin d'améliorer les conditions d'accueil, simplifier les démarches administratives, modifier les heures d'ouverture ou former les agents (CGP, 1993 ; Cahiers du CR-DSU, 1997), avec une importance particulière accordée à l'accueil des populations d'origine étrangères (Barou et al., 1994 ; Fourier et al., 1996).

Les services publics municipaux avaient certainement joué un rôle pilote dans le processus de rapprochement avec les populations (CNFPT et CNDSQ, 1987 ; CNFPT et DIV , 1992). Mais le thème de la proximité finit par s'imposer comme mot d'ordre national en direction des services de l'État. À tel point que plusieurs administrations régaliennes ont entrepris de se réformer sous le sceau de la proximité. Ainsi, après avoir mis en place, en 1990, les premières « Maisons de la justice et du droit » dans les quartiers « sensibles » (Ministère de la Justice, DIV, 1992), l'expérience a été consacrée par la loi du 18 décembre 1998 (sur le thème de la «justice de proximité », cf. (Wyvekens, 1998); pour une synthèse des connaissances, cf. (Peyrat, 2002)). Précédées par d'infructueuses tentatives de développer l'îlotage (Monjardet, 1991 ; CNV, 1992), les réflexions sur la «police de proximité » ont été amorcées à l'aube des années 1990 (Jankowski, 1992), avant que le concept reçoive une consécration officielle sous le ministère Chevènement (Ferret et Ocqueteau, 1998 ; ENA, 2000).

L'une des principales innovations portée par la politique de la ville, dans les années 1990, est sans doute le développement de la «polyvalence » entendue comme la mise en commun de moyens et la coopération entre divers services. La polyvalence n'est pas spécifique aux quartiers urbains ; les expérimentations en milieu rural les avaient devancés (des expériences de polyvalence postale ont été menées dès les années 1960 et la polyvalence est au cœur de la politique d'aménagement du territoire à partir du début des années 1990). Vis-à-vis des zones urbaines défavorisées, la démarche ne prend réellement son essor qu'à la suite d'un appel national à projets lancé en 1996, dans le cadre du Pacte de relance pour la ville, pour promouvoir les «plates-formes de services publics». L'État s'efforcera par la suite de rationaliser les diverses expériences de 
polyvalence, rurales et urbaines, autour du concept fédérateur de «maisons des services publics », auxquelles la loi Voynet sur l'aménagement du territoire (1999) a fourni une assise législative ${ }^{42}$.

Si la proximité revient toujours à agir «au plus près de l'usager» en se rapprochant physiquement de lui, sa visée demeure équivoque dans les discours et la stratégie de la politique de la ville. Dans une première orientation, la proximité est pensée comme un recentrage sur le «service rendu» ou le «service du public » (CNPFT et DIV, 1992 ; DIV, 1995 ; DGAFP, 1998b). Elle trouve alors son fondement théorique dans un changement de paradigme qui verrait le passage d'une « logique d'équipement » à une «logique de services », où l'enjeu serait moins l'affirmation de la puissance publique que la facilitation de la vie des habitants affectés par l'exclusion (Béhar et Estèbe, 1995). Mais une seconde orientation semble plus soucieuse de restaurer les symboles de la puissance publique. Elle a été officiellement formulée en ces termes ; «les services publics remplissent une fonction symbolique ; ils témoignent du pacte républicain qui lie les individus, les groupes sociaux et la communauté nationale »(DIV, 1995). Là où les décideurs ne voient pas de contradiction (ce dernier document de la DIV développe en fait les deux orientations), certains chercheurs estiment que cette fonction symbolique ne s'accompagne pas toujours, en pratique, d'une réelle amélioration du service rendu aux habitants (Bonetti, 1995 ; Béhar et Gorgeon, 1998).

La logique de proximité se trouve également prise en tension avec l'objectif d'accessibilité. Des chercheurs ont pointé les possibles écueils d'une stratégie qui ferait de la proximité une fin en soi (Ghorra-Gobin et Kirszbaum, 2001). Le rapprochement des services avec le terrain serait sous-tendu par l'hypothèse contestable d'une proximité physique garante d'une meilleure accessibilité (Béhar, 1995a), une offre de services trop abondante dans les quartiers risquant au contraire d'accentuer le sentiment de captivité des habitants (Bavoux, 1997). Ces interrogations ont commencé de transparaître quand l'enjeu la politique de la ville

42 Outre celui de l'Aménagement du territoire, le ministère de la Fonction publique s'est également saisi de cette question. Cf. (Leroy, 1998 ; Dire, 1999). 
a été défini comme celui du désenclavement des quartiers et de leurs liaisons avec le reste de la ville. Mais le discours officiel a constamment oscillé, depuis lors, entre l'affirmation d'un objectif «d'accessibilité à la ville» et une finalité concurrente, résumée dans cette formule: créer « des quartiers comme les autres », c'est-à-dire équipés comme les autres. Ce n'est qu'à la fin des années 1990 que des voix officielles ont commencé à pointer les limites de la proximité, pour poser cette question : «enfermement ou tremplin ?»(DIV, 2000). Mais le centre de gravité des réflexions sur l'accessibilité à la ville se situe dans les administrations proprement « urbaines » (ministère de l'Équipement et RATP) qui ont soutenu d'innombrables recherches sur ce thème, en particulier dans le domaine des transports, plutôt qu'au sein de la Délégation interministérielle à la ville qui pense davantage la question des services publics dans une perspective sociale et citoyenne.

\subsection{Les médiations entre fonctions d'urbanité et d'ordre public}

Le développement de fonctions de médiation, à l'interface des services publics et des usagers urbains, constitue le volet « relationnel » de la logique de proximité et incarne, à ce titre, une autre facette du débat sur la territorialisation de l'action publique. Autant le rapprochement physique des équipements peut se comprendre comme une tentative d'abolir les distances spatiales, autant les médiations s'efforcent de résorber la distance sociale entre habitants des quartiers «sensibles » et institutions, afin de mieux coordonner leurs relations. Mais le concept de médiation est très peu homogène dans ses constructions doctrinales comme dans ses dispositifs pratiques ${ }^{43}$.

Si l'on met de côté la dimension proprement juridique de la médiation, comprise comme un mode de résolution des litiges, cette pratique renvoie à deux logiques très différentes dans les territoires urbains: elle peut recouvrir des

43 Pour une tentative de clarification conceptuelle, cf. par exemple (Briant et Palau, 1999; Bonafé-Schmitt et al., 1999) ; pour une mise en perspective européenne, cf. (Wieviorka, 2002), qui rend compte d'un séminaire international organisé sur ce sujet par le ministère de la Ville. 
initiatives de la société civile appelées à former des corps intermédiaires entre les habitants et institutions, ou se voir au contraire instrumentée par ces dernières dans un but de reconquête des territoires sensibles. Le premier sens a longtemps prévalu dans la politique de la ville quand les associations d'habitants, prenant appui sur des chefs de projets immergés dans la communauté du quartier ou sur des universitaires investis dans des recherches-actions, ont pu apparaître comme les fers de lance de l'innovation urbaine dans les domaines de l'école (APU, 1983), du logement (Anselme et al. 1987), de la justice (Dourlens et VidalNaquet, 1993) ou de l'insertion économique (Jaillet, 1993). Ces pratiques n'ont pas manqué de théoriciens, du Cerfi au Cerfise, du CSTB aux tenants de «l'économie solidaire », tous ayant bénéficié des commandes du ministère de l'Équipement. Parmi ces pratiques urbaines qui en appellent à l'implication active des usagers, les « régies de quartier » sont celles qui ont fait l'objet de l'attention la plus soutenue ${ }^{44}$.

Ce n'est pourtant pas dans cette production «communautaire » des services que les médiations ont connu leur essor le plus spectaculaire, mais dans la nébuleuse des «métiers de la ville » qui ont fortement inspiré la mise en place du dispositif national des «emplois jeunes » à partir de 1998. Si, à l'inverse des initiatives de la société civile, ces fonctions de médiation sont des créations institutionnelles, leur officialisation procède d'une capitalisation des expérimentations antérieures. Avant la mise en place des emplois jeunes, des chercheurs avaient ainsi commencé d'étudier ces nouveaux métiers, qu'il s'agisse des «grands frères» (Duret, 1995), des «agents d'ambiance» (Bourgeois et Paquiet, 1996) des «femmes relais» (Delcroix et al., 1996) ou des « correspondants de nuit» (Dartiguenave et Réto, 1998).

Le programme emplois jeunes a suscité une abondante littérature, où se mêlent les points de vue de responsables publics et de chercheurs sollicités pour théoriser leurs fonctions (Heurgon et Stathopoulos, 1999 ; Brévan-Picard, 2000, tome 2). Un premier registre d'analyse a vu dans les médiateurs des «agents d'urbanité »

44 Pour une présentation des principales recherches conduites sur ce thème, cf. (Plan urbain, 1994). 
(Heurgon, 2001) susceptibles d'inverser la logique rationalisatrice des services publics, qui avait abouti à supprimer nombre de fonctions « relationnelles », par exemple les receveurs des bus (Godard, 1999). Ces agents permettraient aux services de reprendre pied sur des territoires délaissés où ils créeraient une interface, ouvriraient un espace de partenariat avec les usagers selon une logique du «coproduire » nécessaire à la fabrication du lien social et de l'urbanité (Jacquier, 1999). Dans cette optique, la médiation est regardée comme un «laboratoire social » et une «opportunité de modernisation du service public », pour reprendre certaines expressions du rapport Brévan-Picard. L'enjeu d'avenir serait celui de la consolidation, ce qu'expriment certains termes récurrents : « professionnalisation », « qualification », « pérennisation »...

Des chercheurs portent un regard beaucoup plus critique sur ces métiers. Ils s'interrogent tout d'abord sur la nature et la qualité des recrutements, la proximité des médiateurs avec la population des «quartiers » faisant jouer des critères ethniques que le modèle républicain réprouve officiellement (Joseph, 1998). La position de ces auxiliaires du service public vis-à-vis d'institutions, dont ils prolongent et adaptent l'activité sur des territoires identifiés comme problématiques, est à la source d'autres interrogations. Les médiations se feraient à sens unique, sans toucher au cœur du fonctionnement institutionnel ${ }^{45}$. Une occasion aurait ainsi été manquée de «qualifier mutuellement» les exclus et les institutions (Rosenberg, 1998). Différentes études soulignent aussi l'ambivalence de ces nouvelles fonctions, partagées entre utilité sociale et instrumentalisation à des fins de pacification des territoires «sensibles » (Ocqueteau, 1999). À propos des aides éducateurs, l'objectif de « surveillance communautaire immédiate » et «ethnique» aurait prévalu sur celui de citoyenneté (Charlot et al., 2000). Il faudrait ainsi reconnaître l'avènement de dispositifs de «traitement parainstitutionnel du désordre » (Macé, 1997a) ou de «professionnels de l'ordre en public » (Roché, 1999).

45 Cf. (Gorgeon, 2000) à propos des ADS (adjoints de sécurité) dans la police nationale, et (Faget, 2001), à propos des ALMS (agents locaux de médiation sociale). 


\subsection{Le traitement des quartiers pauvres entre « spécificité » et «droit commun »}

À quelle norme d'administration les pouvoirs publics se réfèrent-ils quand ils affirment vouloir rapprocher la condition des quartiers pauvres de celle des autres quartiers ? La politique de la ville est traversée par une ambiguïté constitutive, entre le besoin de spécifier des normes d'intervention pour tenir compte de la situation particulière de ces quartiers, et la volonté d'y appliquer un traitement de « droit commun », afin d'éviter de les particulariser davantage ${ }^{46}$.

Dans ses premiers développements, cette politique a privilégié l'invention de normes nouvelles et spécifiques de délivrance des services, en appui sur la participation des habitants. Cette approche a trouvé ses justifications sociologiques dans l'éloge des «quartiers populaires» (Laé, 1991) ou de «l'intégration communautaire »(Genestier, 1991). Le début des années 1990 signale toutefois un déplacement des priorités de la politique de la ville qui, entrée en «maturité », renonce à la tentation de l'expérimentalisme permanent pour en appeler à la mobilisation des politiques sectorielles dites de «droit commun ». Dès lors, le débat se recentre sur le degré admissible d'adaptation de ces normes du droit commun. Ce débat s'est cristallisé dans l'opposition entre deux logiques, celle des contrats de ville, chargés d'infléchir les normes de l'action publique, non seulement dans les quartiers «prioritaires », mais à l'échelle des agglomérations, afin de renforcer leur intégration urbaine, et celle du Pacte de relance pour la ville instituant le principe d'un traitement dérogatoire réservé à ces seuls quartiers. Face à la difficulté de promouvoir des normes spécifiques sans trop les éloigner du droit commun, la suppression de la politique de la ville fut un temps envisagée par le ministère Aubry (1997). Mais cette solution fut abandonnée au profit d'une refondation de la doctrine d'intervention des services publics en direction de ces quartiers. Dans l'esprit de la loi de lutte contre les exclusions du 29 juillet 1998,

\footnotetext{
46 Sur cette tension entre « exception» et «droit commun» dans la politique de la ville, cf. (Profession banlieue, 1998).
} 
la commission Cavallier s'est attelée à cette tâche ${ }^{47}$. La loi contre les exclusions, explique son rapport (Cavallier, 1999), vise « un objectif général de retour au droit commun » et ne construit pas de «systèmes spécifiques de prise en charge » des pauvres ; elle s'attache au contraire à «préciser les modalités d'accès à des droits déjà reconnus de façon à les rendre effectifs ». Les contrats de ville, ajoute le rapport, sont les vecteurs privilégiés de la mise en œuvre des dispositions de la loi contre les exclusions, et devront à l'avenir interroger ce qui, dans le fonctionnement ordinaire des prestations de droit commun, provoque des évictions et mises à l'écart, pour « dépasser la logique de fourniture de prestations spécifiques au profit de véritables fonctions collectives d'intérêt public », garants de la qualité du service rendu.

Cette « réinvention d'un droit commun » qui « exige d'avancer sur deux fronts, celui de la singularité et celui de l'universalité », selon l'expression de Daniel Béhar (Béhar, 1995b), l'un des principaux inspirateurs de cette reformulation normative, laisse toutefois en suspens la question du devenir des quartiers populaires. Car un autre courant d'inspiration de la politique de la ville a fini par acquérir une influence prépondérante. Ce courant prend naissance dans les années 1980 avec le dispositif «Banlieues 89 », puis s'incarne dans différents dispositifs : la Loi d'orientation pour la ville dite LOV (1991), les Grands projets urbains (1992), les Grands projets de ville (1999) et, récemment, dans la loi sur la Rénovation urbaine (2003). Faisant une large place aux démolitions, ces dispositifs affichent parfois explicitement le but «d'en finir avec les grands ensembles » (Banlieues 89, 1991) ou de « casser les ghettos» (Bartolone, 1999). Leur ambition tient moins dans le «retour du droit commun» que dans la réintégration de ces quartiers et de leurs habitants dans «le droit commun ». Il s'agit de banaliser ces territoires, conçus selon une logique de monofonctionnalité résidentielle, en les dotant de l'ensemble des attributs constitutifs d'un mode de vie urbain «normal », à commencer par un niveau d'équipements et services que l'on devrait trouver dans tout quartier «normal». En filigrane de cet idéal

${ }^{47}$ La Commission Cavallier était chargée de formuler des recommandations pour la négociation des contrats de ville du XII ${ }^{\mathrm{e}}$ Plan (2000-2006). 
normatif d'une ville homogène, une intention est perceptible, celle de restaurer le «pacte républicain» mis en péril par la concentration spatiale de populations d'origine immigrée. Peu de chercheurs soutiennent cette orientation. Elle fut même très contestée lors d'un séminaire «chercheurs-décideurs » organisé parallèlement à l'élaboration de la Loi d'orientation pour la ville (Rudder et Garin-Ferraz, 1991).

\section{Conclusion}

Peut-on parler d'une «pensée publique» au terme de cette revue de la littérature sur les services publics et la ville ? Sur cet objet très peu monolithique, les réflexions qui ont été présentées procèdent d'un entrelacs de travaux hétéroclites, plus ou moins soumis aux vicissitudes de la commande publique, laquelle porte avant tout témoignage des transformations et des hésitations de l'État face aux mutations sociales et urbaines. S'ajoutent des effets de mode et emprunts internationaux qui rendaient nécessaire une mise en perspective de la production des années 1990 .

Aussi divers soit-il, le corpus rassemblé suggère néanmoins un enchaînement causal entre les trois figures identifiées: la diversité accrue des conditions territoriales de production des services aurait non seulement conduit à l'éclatement du modèle unitaire du « service public à la française », mais renforcé les tendances à l'éclatement de la ville ; les services publics subiraient ainsi les conséquences de phénomènes qu'ils ont contribué à aggraver par leur soumission à des principes de - le marché, le local - vecteurs de dissociation entre territoires et de ségrégation entre populations ; les services publics sont dès lors invités à se rapprocher des quartiers en difficulté et à établir un compromis entre les exigences de compétitivité et de justice sociale. Cette thèse paraît cependant trop généralisante, car elle repose sur une idéalisation rétrospective du modèle ancien et ne prend pas toujours en compte le caractère profondément hétérogène du «service public à la française ». Surtout, selon le diagnostic qu'elles 
formulent, les réponses à la crise simultanée des services publics et de la ville oscillent entre deux solutions difficilement conciliables : le souci « républicain» de restaurer l'ordre ancien et la recherche d'un ajustement pragmatique au nouvel ordre social et urbain.

\section{Références}

Affichard, J. (Eds.), 1994. Décentralisation et coordination. Les principaux modèles d'analyse. Actes des journées du 8 et 9 mars, Institut international de Paris, ministère de l'Équipement, ministère de l'Environnement, La Défense.

Affichard, J., 1995. Management public et traitement des usagers. In: David A., et al. (Eds.), Le service public ? La voie moderne. Colloque de Cerisy. L'Harmattan, Paris, pp. 281-294.

Affichard, J., De Foucault, J.B. (Eds.), 1992. Justice sociale et inégalités. Esprit, Paris.

AJDA (Actualité juridique. Droit administratif), 1997. Le service public. Unité et diversité 20 .

Allen, B., Conan, M., 1987. Évaluer et améliorer les services publics. Analyses et propositions à partir d'exemples en Amérique et en Europe du Nord. Rapport de recherche, Plan urbain, ministère de l'Équipement.

Annales de la recherche urbaine, 1997. Emplois du temps 77.

Annales de la recherche urbaine, 2000. Nuits et lumières 87.

Annales des mines, réalités industrielles, 1991. L'Europe des grands réseaux.

Annales des mines, réalités industrielles, 1994. Les réseaux de services publics.

Anselme, M., et al., 1987. Entre logeurs et logés. Rapport de recherche, Cerfise, ministère de l'Équipement.

APU (Atelier populaire d'urbanisme), 1983. Participation des habitants à la conception d'un CES adapté à leur quartier. Rapport de recherche, Plan urbain, ministère de l'Équipement. 
ARSS (Actes de la recherche en sciences sociales), 1998. De l'état social à l'état pénal 124.

Ascher, F., 1995. Métapolis ou l'avenir des villes. Odile Jacob, Paris.

Ascher, F. (Ed.), 1995. Le logement en questions. L'habitat dans les années quatre-vingt-dix ; continuité et ruptures. CSTB, Plan construction et architecture. Éditions de l'Aube, La Tour d'Aigues.

Ascher, F., 1998, La République contre la ville. Essai sur l'avenir de la France urbaine. Éditions de l'Aube, La Tour d'Aigues.

Atger, C., et al., 2002. L'enquête publique des plans de déplacements urbains. Enquête publique et concertation. Quelques recommandations. Certu, ministère de l'Équipement, Lyon.

Attias, D., 1997. Concurrence et régulation des services publics. Comparaison internationale. Rapport, Commissariat général du plan. La Documentation française, Paris.

Auby, J.F., 1982. Les services publics locaux. Que sais-je ? Puf, Paris.

Bachelet, F., Rangeon, F., 1996. La politique de la ville ou les difficultés de l'interministérialité locale. Politiques et management public 14 (3).

Ballain, R., Béhar, D., 1998. Vingt ans après la réforme de 1977, refonder la politique du logement. Fondations 6, 51-66.

Ballion, R., 1982. Les consommateurs d'école. Stratégies éducatives des familles. Stock, Paris.

Bandt, J. (de), Gadrey, J. (Eds.), 1994. Relations de service, marchés de services. CNRS Éditions, Paris.

Banlieues 89, 1991. Pour en finir avec les grands ensembles. Assises de Bron, 4 et 5 décembre 1990. DIV, Paris.

Barou, J., et al., 1994. L'accueil à l'épreuve de l'intégration. Bureaux d'accueil, usagers et services publics. Rapport de recherche, ISM-Rhône-Alpes.

Barthélemy, J.R., Leflaive, M., 1996. Fiscalité et tarifs locaux, effets sur une longue période. Le cas de l'agglomération bordelaise. Rapport de recherche, Programme de recherche «La ville éclatée, enjeux et conditions d'une régulation 
économique, sociale et territoriale », Fondation des villes, DAEI, DRAST, ministère de l'Équipement.

Bartolone, C., 1999. Discours. Rencontres nationales des GPV, Vaulx-en-Velin, 10 décembre.

Bataille, C., 1999. Modernisation et développement du service public de l'électricité. Rapport fait au nom de l'Assemblée nationale le 4 février.

Baufils, M.L., et al., 1999. Aménager la ville demain. Une action collective. Éditions de l'Aube. La Tour d'Aigues.

Bautier, E., et al., 1995. Travailler en banlieue. La culture de la professionnalisé. L'Harmattan, Paris.

Bavoux, P., 1997. Des démarches territorialisées de services publics impulsées par l'État. Bref historique. Les cahiers du CR-DSU 17, 6-7.

Béhar, D., 1995a. La question sociale et les services au public. In: DIV, Politique de la ville. Les services au public dans les quartiers. Actes du colloque du 29 juin, La Plaine-Saint-Denis, pp. 15-18.

Béhar, D., 1995b. Banlieues ghettos, quartiers populaires ou ville éclatée ? Annales de la recherche urbaine 68-69, 6-14.

Béhar, D., 1999. Les DDE et les politiques d'aménagement. De la connaissance sur les territoires à la territorialisation de l'action de l'État. Une lecture transversale des évaluations locales. Rapport de recherche, Acadie, Plan urbain, ministère de l'Équipement.

Béhar, D., Estèbe, P., 1995. La puissance publique et le lien social. In: Plan urbain, services urbains et gestion locale. Enjeux et perspectives de recherches 1985-1993. Ministère de l'Équipement, pp. 381-400.

Béhar, D., Estèbe, P., 1996. Le pacte de relance pour la ville. Esprit 219.

Béhar, D., Gorgeon, C., 1998. Évaluation de l'appel à projets plates-formes de services publics. Rapport de recherche, Acadie, DIV.

Bengaouer, M., Pestre-Mazières, M., 1999. Les services publics en milieu urbain. Synthèse des diagnostics établis par les préfets. Rapport de l'IGA au ministère de l'Intérieur et à la Délégation interministérielle à la ville. 
Bergougnoux, J., 2000. Services publics en réseau. Perspectives de concurrence et nouvelles régulations. Rapport, Commissariat général du plan. La Documentation française, Paris.

Berland, J.M., et al., 1995. La place des usagers dans la gestion et dans la régulation des réseaux urbains. Rapport de recherche, ENPC, LATTS, Plan urbain, ministère de l'Équipement.

Bezès, P., 2002. La construction historique des politiques de réforme de l'administration en France depuis les années 1960. L'hypothèse d'un processus de différenciation intra-étatique. Communication au VII ${ }^{\mathrm{e}}$ congrès de l'Association française de science politique, Lille, 18 au 21 septembre.

Bieber, A., et al., 1993. Questions vives pour une prospective de la mobilité quotidienne. Collection Synthèse Inrets 19.

Blanc, C., 1993. Pour un État stratège, garant de l'intérêt général. Rapport, Commissariat général du plan. La Documentation française, Paris.

Bodiguel, J.L., 1999. Rapport général de synthèse. In: Pauliat, H. (Ed.), La cohésion territoriale et les services publics en Europe. Interprétation et portée de l'article 7D du traité d'Amsterdam. Pulim, Limoges, pp. 195-215.

Boltanski, L. (Ed.), 1992. Innovations et pratiques locales dans l'administration. Rapport de recherche, GSPM/EHESS.

Bonafé-Schmitt, J.P., et al., 1999. Les médiations, la médiation. Erès, Ramonville Saint-Agne.

Bonetti, M., 1995. L'enjeu de la gestion urbaine, Annales de la recherche urbaine $68-69,15-25$.

Bonnemaison, G., 1982. Face à la délinquance, prévention, répression, solidarité. Rapport au Premier ministre. La Documentation française, Paris.

Bonvalet, C., Fribourg, A.M. (Eds.), 1990. Stratégies résidentielles. Actes du séminaire, Ined, Plan construction et architecture, ministère de l'Équipement Bourdieu, P. (Ed.), 1993. La misère du monde. Seuil, Paris.

Bourgeois, F., Paquiet P., 1999. Agents d'ambiance dans les transports publics urbains. Pratiques, effets et enjeux d'un métier en émergence. Rapport de recherche, économie et humanisme, Predit Drast, ministère de l'Équipement. 
Bouveau, P., 1997. L'école à l'ère des ZEP. Annales de la recherche urbaine 75, $33-42$.

Bravo, J., 1999. Rapport final de l'Instance d'évaluation de la politique de la ville en Île-de-France. Conseil régional d'île-de-France, Préfecture de région d'Île-deFrance.

Brévan, C., Picard, P., 2000. Une nouvelle ambition pour les villes. De nouvelles frontières pour les métiers. Rapport au ministre délégué à la Ville.

Briant, V. de, Palau Y., 1999. La médiation : définition, pratiques et perspectives. Nathan, Paris.

Bricq, N., 1999. Mission de réflexion et de concertation sur la transposition de la directive européenne sur le marché intérieur du gaz. Rapport au Premier ministre. Brouant, J.P., Jégouzo, Y., 1998. La territorialisation des politiques et du droit de l'habitat social. Les cahiers du Gridauh 2.

Brun, G., Faivre d'Arcier, B. (Eds.), 1993. La complémentarité entre la voiture particulière et les transports collectifs en zone urbaine. Rapport du groupe de travail du Conseil national des transports.

Burland, A., Laufer, R., 1980. Management public. Gestion et légitimité. Dalloz, Paris.

Cahiers du CNFPT, 1987. Collectivités locales et management 22.

Cahiers du CR-DSU, 1997. Les services publics. Entre adaptation et territorialisation 17.

Cahiers du génie urbain, 1996. Demain, les services urbains 13.

Carrère, G., 1991. La formation des fonctionnaires de l'État et la politique de la ville. Rapport au ministre de la Ville.

Cascalès, M., 1997. La reconnaissance des échelons territoriaux dans la réforme de l'État. Revue administrative 297, 321-326.

Cavallier, G., 1999. Nouvelles recommandations pour la négociation des contrats de ville de la nouvelle génération (2000-2006). Rapport au ministre délégué à la Ville.

Cerfi, 1973. Les équipements du pouvoir. Généalogie du capital. Recherches 13. 
Certu, 2000. Concertation et débat public. Actes du colloque de Lyon, 14 juin, ministère de l'Équipement.

CGP (Commissariat général du plan), 1993. Les services publics et l'accueil des populations défavorisées. Évaluation de la politique d'accueil. Rapport. La Documentation française, Paris.

Chalas Y., Dubois-Taine, G. (Eds.), 1997. La ville émergente. Éditions de l'Aube, La Tour d'Aigues.

Champion, J.B., Tabard, N., 1996. Les territoires de l'école publique et de l'école privée. Économie et statistiques 293.

Charlot, B., 1994. La territorialisation des politiques éducatives. Une politique nationale. In: Charlot, B. (Ed.), École et territoire. Nouveaux espaces, nouveaux enjeux. Armand Colin, Paris.

Charlot, B., Emin, J.C. (Eds.), 1997. Violences à l'école. État des savoirs. Armand Colin, Paris.

Charlot, B., et al., 2000. Les aides éducateurs. Le lien social au détriment de la citoyenneté. Les cahiers de la sécurité intérieure 40, 47-63

Chatelus, G., Perrot, J.Y. (Eds.), 2000. Financement des infrastructures et des services collectifs ; le recours au partenariat public-privé. Les enseignements des expériences françaises dans le monde. Presses de l'ENPC, Paris.

Chaty, L., 1997. L'administration face au changement. Projets de services et centres de responsabilité dans l'administration française. L'Harmattan, Paris.

Chausseron, C., 2001. Le choix de l'établissement au début des études secondaires. DPD, ministère de l'Éducation nationale. Note d'information 142.

Chauvière, M., Godbout, J. (Eds.), 1992. Les usagers entre marché et citoyenneté. L'Harmattan, Paris.

Chevallier, J., 1992. Regards sur l'administré. In: Chauvière, M., Godbout, J. (Eds.), Les usagers entre marché et citoyenneté. L'Harmattan, Paris, pp. 25-40. Clark, T., et al., 1985. L'innovation municipale à l'épreuve de l'austérité budgétaire. Rationalisation des services publics et contraintes politiques. Rapport de recherche, Plan urbain, ministère de l'Équipement. 
Closets, F. (de), 1989. Le pari de la responsabilité. Rapport, Commissariat général du plan. La Documentation française, Paris.

CNFPT (Conseil national de la fonction publique territoriale), CNDSQ (Commission nationale pour le développement social des quartiers), 1987. Services municipaux et développement social des quartiers. Compte rendu du séminaire organisé à Lyon les 18 et 19 novembre 1986.

CNFPT (Conseil national de la fonction publique territoriale), DIV (Délégation interministérielle à la ville), 1992. Services des villes et développement social urbain. Compte rendu du séminaire organisé à Angers les 15 et 16 novembre. Éditions du CNFPT, Paris.

CNV (Conseil national des villes), 1992. Bilan des 64 propositions du rapport de la Commission des maires sur la sécurité.

CNV (Conseil national des villes), 1998. Rapport 1994-1997. La Documentation française, Paris.

Coing H., 1998. Services urbains et ville. Nouveaux enjeux. Sociétés contemporaines $32,57-69$.

Coloos, B., 1995. La production et la gestion de l'habitat. In: Ascher, F. (Ed.), Le logement en questions. L'habitat dans les années quatre-vingt-dix. Continuité et ruptures. Éditions de l'Aube, La Tour d'Aigues, pp. 69-107.

Commaille, J., 2000. Territoires de justice. Une sociologie politique de la carte judiciaire. GIP « Droit et justice ». Puf, Paris.

Conan, M., 1988. Gestion dynamique de la productivité des services urbains. Enseignement de vingt-deux ans d'expérience de gestion et d'évaluation dans la municipalité de New York, 1965-1987. CSTB, Plan urbain, ministère de l'Équipement.

Conseil d'État, 1994. Service public, services publics. Déclin ou renouveau. Études \& documents 46. La Documentation française, Paris.

Conseil d'État, 1997. Sur le principe d'égalité. Études \& documents 48. La Documentation française, Paris.

Conseil d'État, 1999. L’intérêt général. Études \& documents 50. La Documentation française, Paris. 
Corcuff, P., Lafaye, C., 1993. Les dilemmes de l'équilibre social. Une innovation locale dans le secteur HLM. Revue française des affaires sociales 47 (3), 63-80. Corcuff, P., Lafaye, C., 1996. Tensions et redéfinitions dans une DDE. In: Grémion, C., Fraisse, R. (Eds.), Le service public en recherche. Quelle modernisation ? La Documentation française, Paris, pp. 285-293.

Cour des comptes, 2002. La politique de la ville. Rapport public.

Crif (Conseil régional d'Île-de-France), Prif (Préfecture de région d'île-deFrance), 1999. Adaptation des services publics. Dossier thématique de l'Instance d'évaluation de la politique de la ville en Île-de-France.

Crozier, M., Thoenig, J.C., 1975. La régulation des systèmes organisés complexes. Revue française de sociologie 16 (1), 3-32.

DAEI (Direction des affaires économiques et internationales), 1994. Séminaire sur la gestion et le financement privés des infrastructures publiques. Ministère de l’Équipement, Paris-La-Défense, 21-22 novembre.

Damamme, D., Jobert, B., 1995. La ville ou l'injonction contradictoire en politique. Revue française de science politique 1, 3-30.

Dartiguenave, J.Y., Réto, N., 1998. Les correspondants de nuit. L'émergence d'une profession incertaine. Lares, Optima, Rennes.

Davezies, L., Prud'homme, R., 1993. L'économie du partenariat public/privé. In: Martinand C. (Ed.), L'expérience française du financement privé des équipements publics. Économica, Paris.

Dear, M., 1993. Le syndrome Nimby. 2001 Plus/Veille internationale (27), Drast, ministère de l'Équipement.

Debarbieux, E., 1996. La violence en milieu scolaire. 1. État des lieux. ESF, Paris.

Delarue, J.M., 1991. Banlieues en difficultés. La relégation. Syros, Paris.

Delaunay, B., 1993. L'amélioration des rapports entre l'administration et les administrés. Contribution à l'étude des réformes administratives entreprises depuis 1945. LGDJ, Paris. 
Delcroix, C., et al., 1996. Médiatrices dans les quartiers fragilisés. Le lien. Rapport, ministère du Travail et des Affaires sociales. La Documentation française, Paris.

Derouet, J.L., 1992. Le cas des zones d'éducation prioritaires dans l'Éducation Nationale. In: Boltanski, L. (Ed.), Innovations et pratiques locales dans l'administration. Rapport, GSPM, EHESS .

Derouet, J.L., Dutercq, Y., 1990. Consommateurs d'école et modernisation du service public. Les conflits entre parents et établissement scolaire peuvent-ils se construire en cause collective ? Inrep, Paris.

DGAFP (Direction générale de l'administration et de la fonction publique), 1998a. Réformer les administrations. Le dilemme entre unité et diversité. La Documentation française, Paris.

DGAFP (Direction générale de l'administration et de la fonction publique), 1998b. Améliorer la qualité du service rendu à l'usager. Ministère de la Fonction publique.

Delevoye, J.P., 1997. Cohésion sociale et territoires. Rapport, Commissariat général du plan. La Documentation française, Paris.

Denoix de Saint Marc, 1996. Le service public. Rapport au Premier ministre. La Documentation française, Paris.

Dire (Délégation interministérielle à la réforme de l'État), 1999. Les maisons des services publics. Compte rendu d'enquête.

DIV (Délégation interministérielle à la ville), 1993. Les contrats de ville du $\mathrm{XI}^{\mathrm{e}}$ Plan. Dossier-ressources.

DIV (Délégation interministérielle à la ville), 1995. Politique de la ville. Les services au public dans les quartiers. Synthèse des journées thématiques de la ville, cycle 1994-1995.

DIV (Délégation interministérielle à la ville), 1999. Prévention et sécurité. Agir au quotidien dans les villes. Rencontres nationales des acteurs de la ville, Montpellier, 17 et 18 mars. Les éditions de la DIV. 
DIV (Délégation interministérielle à la Ville), 2000. Territoire et proximité. Moderniser le service public des villes. Rencontres de Montreuil des 24 et 25 février. Les éditions de la DIV.

Donzelot, J., 1986. D'une modernisation l'autre. Esprit 8-9, 30-45.

Donzelot, J., Estèbe, P., 1992. Le développement urbain. Constitution d'une politique (1982-1992). Rapport. Comité national d'évaluation de la politique de la ville, DIV.

Donzelot, J., Estèbe, P., 1994. L'État animateur. Essai sur la politique de la ville. Éditions Esprit, Paris.

Donzelot, J., Estèbe, P., 1998. Réévaluer la politique de la ville. Pouvoirs locaux $37,51-60$.

Dossiers d'éducation \& formations, 1997. Violence à l'école. État des recherches. Actes du symposium organisé les 26 et 27 juin, (95). DEP, Ihesi.

Dourlens, C., 1996. L'administration de l'équipement et le secteur associatif. Rapport de recherche, Cerpe, Plan urbain, ministère de l'Équipement.

Dourlens, C., Vidal-Naquet, P., 1993. L'autorité comme prestation. La justice et la police dans la politique de la ville. Rapport de recherche, Cerpe, Commissariat général du plan, DIV, Ihesi, Plan urbain.

Drouet, D., et al., 1996. Ville éclatée et réseaux urbains. Quel rôle peut jouer la régulation des réseaux techniques urbains pour réduire les phénomènes d'éclatement? Les cas de Newcastle, Francfort et Nantes. Rapport de recherche, MLD-RDI, DRAST, ministère de l'Équipement.

Dubedout, H., 1983. Ensemble, refaire la ville. Rapport au Premier ministre. La Documentation française, Paris.

Dubet, F., 1994. Les mutations scolaires et les violences à l'école. Cahiers de la sécurité intérieure (15), 11-26.

Dubet, F. (Ed.), 1997. École, familles. Le malentendu. Textuel, Paris.

Dubet, F. (Ed.), 1999. Le collège de l'an 2000. Rapport à la ministre déléguée chargée de l'enseignement scolaire. La Documentation française, Paris.

Dubet, F., Martuccelli, D., 1998. Dans quelle société vivons-nous ? Seuil, Paris. 
Duhem, B., et al. (Eds.), 1995. Villes et transports. Actes du séminaire 19911994, Plan urbain, ministère de l'Équipement.

Dupuy, F., Thoenig, J.C., 1985. L'administration en miettes. Fayard, Paris.

Duran, P., Thoenig, J.C., 1996. L'État et la gestion publique territoriale. Revue française de science politique 46 (4), 580-622.

Duret, P., 1995. Les grands frères. Identité et fonctionnalité d'une médiation. Rapport de recherche, Injep.

ENA (École nationale d'administration), 2000. La police de proximité. Une révolution culturelle. Séminaire de questions sociales, promotion Averroès (1998-2000).

Estèbe, P., 2002. L'habitant ou le cher disparu. Disparitions, apparitions et résurgences de l'habitant comme figure de la participation politique en France. Cahiers de la sécurité intérieure 49,151-171.

Estèbe, P., Kirszbaum, T., 1996. L'intercommunalité entre optimum territorial et pouvoir local. Rapport de recherche, Acadie, Plan urbain, ministère de l'Équipement.

Eymard-Duvernay, F., Marchal, E., 1996. Les agents des services publics à l'épreuve des usagers. In: Grémion, C., Fraisse, R. (Eds.), Le service public en recherche. Quelle modernisation? La Documentation française, Paris.

Faget, J. (Ed.), 2001. Les agents locaux de médiation sociale. Rapport de recherche, Ihesi, ministère de l'Intérieur.

Ferret, J., Ocqueteau, F., 1998. Évaluer la police de proximité ? Problèmes, concepts, méthodes. Rapport. La Documentation française, Paris.

Flux, 1995. Le service universel et l'équité territoriale. Jusqu'où ? (22).

Fourier, M., 1996. L'accueil des populations étrangères dans les services publics. Rapport, Adri.

Fraisse, R., Serieyx, H., 1994. L’État dans tous ses projets. Un bilan des projets de service dans l'administration. Rapport au ministre de la Fonction publique, Commissariat général du plan. La Documentation française, Paris.

Gadrey, J., 1990. Rapports sociaux de service. Une autre régulation. Revue économique 41 (1), 49-69. 
Gadrey, J., 1997. Service universel, service d'intérêt général, service public. Un éclairage à partir du cas des télécommunications et du secteur postal. Politiques et management public 15 (2), 42-72.

Gaudin, J.P., 1989. Technopolis. Crises urbaines et innovations municipales. Puf, Paris.

Gaudin, J.P., 1993a. Les nouvelles politiques urbaines, Que sais-je ?, Puf, Paris. Gaudin, J.P., 1993b. L'intérêt général entre le central et le local. Rapport de recherche, Drast, ministère de l'Équipement.

Gaudin, J.P. (Ed.), 1996. La négociation des politiques contractuelles. L'Harmattan, Paris.

Gaudin, J.P., Novarina, G. (Eds.), 1997. Politiques publiques et négociation. Multipolarités, flexibilités, hiérarchies. Quelques courants contemporains de recherche. Caisse des dépôts et consignations. CNRS Éditions, Paris.

Gautrat, J., 1996. Les caisses d'allocations familiales confrontées à l'entrée en masse de nouveaux usagers. In: Grémion, C., Fraisse, R. (Eds.), Le service public en recherche. Quelle modernisation? La Documentation française, Paris, pp. 257-266.

Geindre, F., 1993. Villes, démocratie, solidarité. Le pari d'une politique. Rapport, Commissariat général du plan. La Documentation française, Paris.

Genestier, P., 1991. Pour une intégration communautaire. Esprit 2.

Ghorra-Gobin, C., Kirszbaum, T., 2001. La proximité à l'ère métropolitaine. Les politiques d'accès à l'emploi en France et aux États-Unis. Annales de la recherche urbaine $90,138-147$.

Gibert, P., 1983. Management public. Management de la puissance publique. Institut de management public, Paris.

Gibert P., Thoenig, J.C., 1993. La gestion publique, entre l'apprentissage et l'amnésie. Politiques et management public 11 (1), 3-21.

Gilbert, C., Saez, G., 1982. L'État sans qualités. Puf, Paris.

Gillio, C., Ion, J. (Eds.), 1996. Les associations dans le domaine de l'aménagement, l'urbanisme et le cadre de vie. Séminaire de recherche, 23-24 octobre, Cresal, Plan urbain, ministère de l'Équipement. 
Godard, F., 1999. Les services et métiers de la ville. Tenir la distance. In: Heurgon, E., Stathopoulos, N. (Eds.), Les métiers de la ville. Les nouveaux territoires de l'action collective. Éditions de l'Aube, La Tour d'Aigues, pp. 379397.

Gorgeon, C., 2000. Les adjoints de sécurité dans la police nationale. Les cahiers de la sécurité intérieure 40,65-91.

Grémion, P., 1976. Le pouvoir périphérique. Bureaucrates et notables dans le système politique français. Seuil, Paris.

Grémion, C., 1992. Que reste-t-il des administrations déconcentrées ? In: Muller, P. (Ed.), L'administration française est-elle en crise ? L'Harmattan, Paris.

Grémion, C., Mouhanna, C., 1995. Le sous-préfet à la ville. L’Harmattan, Paris. Guichard, O., 1976. Vivre ensemble. Rapport, Commissariat général du plan. La Documentation française, Paris.

Hatchuel, A., et al., 1993. Innovation de produit et modernité publique. Vers un service public prospecteur. Rapport de recherche, CGS, École des mines de Paris. Hébrard, J., 2002. La mixité sociale à l'école et au collège. Rapport au ministre de l'Éducation nationale.

Henry, C., 1999. Étude comparative des conditions et instruments de régulation économique de services publics en réseaux. Rapport, Commissariat général du plan. La Documentation française, Paris.

Hervé, E., 2001. Temps des villes. Rapport au ministre de l'Emploi et de la Solidarité.

Heurgon, E. (Ed.), 2001. Agents d'urbanité ? De nouveaux partenaires pour la ville. Éditions de l'aube, La Tour d'Aigues.

Heurgon, E., Stathopoulos, N. (Eds.), 1999. Les métiers de la ville. Les nouveaux territoires de l'action collective. Éditions de l'Aube, La Tour d'Aigues.

Hirschmann, A., 1972. Face au déclin des entreprises et des institutions. Éditions ouvrières, Paris.

Husson, M.P., Klein, M., 1991. L'Équipement scolaire. Ordre administratif et mobilité résidentielle. Rapport de recherche, Économie et humanisme, Plan urbain, ministère de l'Équipement. 
Ihesi (Institut des hautes études de la sécurité intérieure), 1998. Guide pratique pour les contrats locaux de sécurité. La Documentation française, Paris.

Informations sociales, 1999. Les institutions face au débordement du social (76). Ion, J., 1997. La fin des militants. Éditions de l'atelier, Paris.

Jacquier, C., 1999. Synthèse. In: Heurgon, E., Stathopoulos, N. (Eds.), Les métiers de la ville. Les nouveaux territoires de l'action collective. Éditions de l'Aube, La Tour d'Aigues, pp. 345-349.

Jaillet, M.C., 1993. L'insertion par l'économique. In: Comité national d'évaluation de la politique de la ville. Les enjeux de l'action. DIV, Plan urbain, Caisse des dépôts et consignations.

Jaillet, M.C., 1999. Peut-on parler de sécession urbaine à propos des villes européennes ? In: Donzelot, J., Jaillet, M.C. (Eds.), La nouvelle question urbaine. Actes du séminaire 1999-2000. Collection Recherches (126), Puca, ministère de l'Équipement, pp. 23-44.

Jankowski, B., 1992. La police de proximité. Regard de la recherche sur un nouveau style de police. Rapport de recherche, Ihesi, ministère de l'Intérieur.

Jeannot, G., 1998. Les usagers du service public. Que sais-je ? Puf, Paris.

Jeannot, G., Joseph, I. (Eds.), 1995. Métiers du public. Les compétences de l'agent et l'espace de l'usager. CNRS Éditions, Paris.

Jeannot, G., Quin, C. (Eds.), 1997. Un service public pour les Européens ? Diversité des traditions et espaces de convergence. Ministère de l'Équipement. La Documentation française, Paris.

Jobert, B., 1992. Usagers et agents du service public. Proposition pour l'approche d'un système complexe. In: Chauvière, M., Godbout, J. (Eds.), Les usagers entre marché et citoyenneté. L'Harmattan, Paris, pp. 41-59.

Jobert, A., 1998. L'aménagement en politique. Ou ce que le «syndrome Nimby » nous dit de l'intérêt général. Politix 42, 67-92.

Jobert, B., Muller, P., 1987. L'État en action. Politiques publiques et corporatismes. Puf, Paris. 
Joseph, I., 1998. Le bien commun des villes. In: Sueur, J.P. (Ed.), Demain la ville. Tome II, Rapport au ministre de la Solidarité et des Affaires sociales, pp. 326348.

Joye, D., 1999. L'habitant-usager-citoyen. Urbanisme 307.

Kaysergruber, D., Strobel, P., 1996. Service public, fin de siècle. Contraintes européennes et défi de la pauvreté. In: Grémion, C., Fraisse, R. (Eds.), Le service public en recherche. Quelle modernisation? La Documentation française, Paris, pp. 21-38.

Kirszbaum, T., 1998. Fonction de coordination des services de l'État et contractualisation dans la politique de la ville. Essai de synthèse des évaluations produites dans le cadre du XI ${ }^{\mathrm{e}}$ Plan. Rapport de recherche, Cedov, Plan urbain, ministère de l'Équipement.

Kirszbaum, T., 2001. Modernisation des services publics et éclatement de la ville sous le regard des chercheurs. Collection Recherches 54, Puca, ministère de l’Équipement.

Laé, J.F., 1991. Entre le faubourg et le HLM. L'éclipse du pauvre. Iresco, Paris. Landrieu, J., 1997. Le logement, une mission de service public ? In: Jeannot, G., Quin, C. (Eds.), Un service public pour les européens ? Diversité des traditions et espaces de convergence. Ministère de l'Équipement. La Documentation française, Paris.

Landrieu, J., 1998. Ville éclatée. De nouvelles perspectives de régulation sociale. In: May, N., et al. (Eds.), La ville éclatée. Éditions de l'Aube, La Tour d'Aigues, pp. 21-58.

Langlais, J.L., et al., 1991. Les services publics de proximité dans les quartiers en difficulté. Rapport de l'Igas et de l'IGA au ministre de la Ville.

Langouët, G., Léger, A., 1987. Parcours scolaires et transferts public-privé. Société française 22, 42-50.

Lapeyronnie, D., 1999. Contre-monde. Imitation, opposition, exclusion. Annales de la recherche urbaine 83-84, 53-58.

Lapierre, U., 2000. Logement et marché. Le principe d'exclusion. Études foncières $88,16-19$. 
Lascoumes, P., Le Bourhis, J.P., 1998. Le bien commun comme construit territorial. Identités d'action et procédures. Politix 42, 37-66.

Lefèvre, C., 1998. Gouvernance, institutions et territoires. Les gouvernements métropolitains dans les pays occidentaux. In: May, N., et al. (Ed.), La ville éclatée. Éditions de l'Aube, La Tour d'Aigues, pp. 277-290.

Lefèvre, C., Offner, J.M. (Eds.), 1990. Les transports urbains en question. Usages, décisions, territoires. Celse, Paris.

Le Galès, P., 1995. Du gouvernement des villes à la gouvernance urbaine. Revue française de sciences politiques 45 (1), 57-95.

Lepetit, B., Pumain, D. (Eds.), 1993. Temporalités urbaines. Économica, Paris.

Leroy, M., 1998. La polyvalence dans les administrations. Rapport, DGAFP. La Documentation française, Paris.

Lévy, F., 1988. Bilan-perspectives des contrats de plan de développement social des quartiers. Rapport, Commissariat général du plan.

Lipsky, M., 1980. Street-level Bureaucracy. Dilemmas of the Individual in Public Services. Russel Sage Foundation, New York.

Lolive, J., 1999. La montée en généralité pour sortir du Nimby. La mobilisation associative contre le TGV Méditerranée. Politix 39, 109-130.

Lorcerie, F., 1998. La coopération des parents et des maîtres. Une approche non psychologique. Ville-école-intégration 114, 20-34.

Lorrain, D., 1989. La montée en puissance des villes. Économie et humanisme $305,6-21$.

Lorrain, D., 1990. Le modèle français de services urbains. Économie et humanisme 312 .

Lorrain, D., 1991. De l'administration républicaine au gouvernement urbain. Sociologie du travail 33 (4), 461-484.

Lorrain, D., 1993. Les services urbains, le marché et le politique. In: Martinand, C. (Ed.), L'expérience française du financement privé des équipements publics. Économica, Paris.

Lorrain, D., 1996. Services publics et participation des citoyens. Annuaire des collectivités locales 16, 189-203. 
Lorrain, D., Stoker G. (Eds.), 1995. La privatisation des services urbains en Europe. La Découverte, Paris.

Macé, E., 1997a. Les contours de la médiation. Institution, conciliation, conformation. À propos d'un dispositif de «médiation» de la RATP. Revue française des affaires sociales 2, 225-244.

Macé, E., 1997b. Service public et banlieues populaires. Une coproduction de l'insécurité. Le cas du réseau bus de la RATP. Sociologie du travail 39 (4), 473 498.

Madiot, Y., 1995. Vers une territorialisation du droit. Revue française de droit administratif $11(5)$.

Maguer, A., Berthet, J.M., 1997. Les agents des services publics dans les quartiers difficiles. Rapport, DGAFP. La Documentation française, Paris.

Marcou, G., et al. (Eds.), 1997. La coopération contractuelle et le gouvernement des villes. L'Harmattan, Paris.

Martinand, C. (Ed.), 1993. L'expérience française du financement privé des équipements publics. Économica, Paris.

Martinand, C., 2001. La maîtrise des services publics urbains organisés en réseaux. Journal officiel, Avis et rapports du CES (8), 3 mai.

May, N., et al. (Eds.), 1998. La ville éclatée. Éditions de l'Aube, La Tour d'Aigues.

Mény, Y., Thoenig, J.C., 1989. Politiques publiques. Puf, Paris.

Meuret, D., et al., 2000. Le choix et l'autonomie des établissements scolaires.

Quels effets? Rapport de recherche, Iredu, CNCRE, ministère de l'Éducation nationale.

Minc, A., 1987. La machine égalitaire. Grasset, Paris.

Minc, A., 1994. La France de l'an 2000. Rapport au Premier ministre, Commissariat général du plan. La Documentation française-Odile Jacob, Paris.

Ministère de l'Équipement, 1999. Service universel, service d'intérêt général. Les approches françaises et britanniques dans le cadre de la construction européenne. Séminaire franco-britannique, DAEI, Drast, ministère de l'Équipement. 
Ministère de la Justice, DIV (Délégation interministérielle à la ville), 1992. La justice au cœur de la cité.

Monjardet, D., 1991. Une mission sur un territoire. De la difficulté des policiers à entrer dans les politiques de prévention de la délinquance. Bulletin, revue du CLCJ (26).

Monjardet, D., 1996. Ce que fait la police. Sociologie de la force publique. La Découverte, Paris.

Monjardet, D., 1999. Réinventer la police urbaine. Le travail policier à la question dans les quartiers. Annales de la recherche urbaine 83-84, 15-22.

Mouhanna, C., 1999. Les services publics et la question jeune. Rapport de recherche, DIV.

Moulin, B. (Ed.), 1997. Multiplication des frontières symboliques. Fragmentation de la ville et des groupes sociaux. Rapport de recherche, Plan urbain, ministère de l’Équipement.

Muller, P., 1992. Le modèle français d'administration face à la constitution d'un espace public européen. In: Muller, P. (Ed.), L’administration française est-elle en crise ? L'Harmattan, Paris.

Nemery, J.C., 1981. De la liberté des communes dans l'aménagement du territoire. LGDJ, Paris.

Nemery, C., Wachter, S. (Eds.), 1994. Gouverner les territoires. Datar. Éditions de l'Aube, La Tour d'Aigues.

Neveu, C. (Ed.), 1999. Espace public et engagement politique. Enjeux et logiques de la citoyenneté locale. L'Harmattan, Paris.

Obadia, A. (Ed.), 1997. Entreprendre la ville. Nouvelles temporalités, nouveaux services. Actes du colloque de Cerisy «Vivre la ville demain. Quels enjeux, quels partenaires ?». Éditions de l'Aube, La Tour d'Aigues.

Oblet, T., 1997. En quête de ville. Politiques urbaines et développement de la démocratie. Thèse de sociologie, université Bordeaux II.

Ocqueteau, F., 1999. Mutations dans le paysage français de la sécurité publique. Annales de la recherche urbaine 83-84, 7-13. 
Offner, J.M., 1995. Intervention. In: Coing, H., Offner, J.M. (Eds.), Privatisation des services urbains. Les notions de régulation et d'équité territoriale dans le contexte des privatisations. Quelles orientations donner à la recherche ? Compte rendu de la journée du Latts, pp. 45-46.

Ortiz, L., 1994. Espace et efficacité de l'action, le mythe de l'optimum dimensionnel. In: Némery, J.C. (Ed.), Le renouveau de l'aménagement du territoire en France et en Europe. Économica, Paris, pp. 183-200.

Pain, J., et al., 1997. Violences à l'école. Allemagne, Angleterre, France. Une étude comparative européenne de douze établissements du deuxième degré. Rapport de recherche, Ihesi, DPD.

Payet, J.P., 1995. Collèges de banlieue. Ethnographie d'un monde scolaire. Méridiens-Klincksieck, Paris.

Pêcheur, B., 1991. Valoriser les hommes et les femmes du service public dans le cadre de la politique de la ville. Rapport au ministre de la Fonction publique.

Pény, A., Wachter, S. (Eds.), 1999. Les vitesses de la ville. Éditions de l'Aube, La Tour d'Aigues.

Pesce, R., 1984. Développement social des quartiers. Bilans et perspectives. 1981-1984. Rapport au Premier ministre. La Documentation française, Paris.

Peyrat, D. (Ed.), 2002. La justice de proximité (special issue). Problèmes politiques et sociaux 869 .

Pharo, P., 1985. Le civisme ordinaire. Méridiens-Klincksieck, Paris.

Picard, P., 1991. L'amélioration du service public dans les quartiers. Rapport au ministre de la Ville.

Picq, J., 1994. L'État en France. Servir une nation ouverte sur le monde. Rapport au Premier ministre. La Documentation française, Paris.

Pisani, E., 1956. Administration de gestion, administration de mission. Revue française de science politique 16 (2).

Plan urbain, RATP, DRI, 1991. La relation de service dans le secteur public. Tome I : Actes du séminaire (1989-1990). Tome II à V : Actes du colloque de janvier 1991 « À quoi servent les usagers ?». 
Plan urbain, 1994. Les régies de quartier. Expérience et développements. Regards de chercheurs. Ministère de l'Équipement. La documentation française, Paris. Plan urbain, 1995. Services urbains et gestion locale. Enjeux et perspectives de recherches. 1985-1993. Ministère de l'Équipement.

Plan urbain, 1997. Qui fait la ville aujourd'hui ? Intervention de la puissance publique dans les processus de production urbaine. Ministère de l'Équipement.

Préteceille, E., et al., 1986. Ségrégation urbaine. Classes sociales et équipements en région parisienne. Anthropos, Paris.

Profession banlieue, 1998. La politique de la ville. Entre exception et droit commun.

Quatrebarbes, B. (de), 1996. Usager ou client ? Marketing et qualité dans les services publics. Éditions d'Organisation, Paris.

Querrien, A., 1999. Pourquoi parler de gouvernance urbaine? In: Heurgon, E., Stathopoulos N. (Eds.), Les métiers de la ville. Les nouveaux territoires de l'action collective. Éditions de l'Aube, La Tour d'Aigues, pp. 175-186.

Quin, C. (Ed.), 1995. L'administration de l'Équipement et ses usagers. Conseil général des ponts et chaussées. La Documentation française, Paris.

Rhein, C., et al., 1996. Division sociale de l'espace et inégalités de scolarisation. Rapport de recherche, CNRS, Plan construction et architecture, ministère de l'Équipement.

Roché, S., 1999. Les règles d'hospitalité et les professionnels de l'ordre en public. Cahiers du CR-DSU (22), 29-33.

Rosenberg, S., 1995. Le service public ou l'état de la société. In: Plan urbain, Services urbains et gestion locale. Enjeux et perspectives de recherches. 19851993. Ministère de l’Équipement, pp. 423-455.

Rosenberg, S., 1997. Évaluation de la politique de la ville en Île-de-France, Groupe Services Publics. La Poste. Rapport de recherche, Conseil régional, Préfecture de région d'Île-de-France.

Rosenberg, S., 1998. Qualification mutuelle des exclus et des institutions. Un mode de reconstruction de la société. Ville-école-intégration (113), 29-43. 
Rosenberg, S., 2000. Agents et bureaux de poste dans les quartiers sensibles. Flux 42.

Rouban, L., 1994. Le pouvoir anonyme. Les mutations de l'État à la française. Presses de la FNSP, Paris.

Rouban, L., 1997. La fin des technocrates ? Presses de la FNSP, Paris.

Rousseau, M.P., 1998. De l'électeur au consommateur de services publics locaux. Urbanisme 299, 83-84.

Rudder, V. (de), Garin-Ferraz, G. (Eds.), 1991. Loi d'orientation pour la ville. Séminaire chercheurs-décideurs. DAU, ministère de l'Équipement.

Sandrier, J.C., 2001. Associations et politique de la ville. Rapport au premier Ministre. La Documentation française, Paris.

Santel, G., 1998. La modernisation de l'administration territoriale de l'État. Rapport au ministre de la Fonction publique.

Sauvez, M., 2001. La ville et l'enjeu du « développement durable ». Rapport au ministre de l'Aménagement du territoire et de l'environnement. La Documentation française, Paris

Schwartz, B., 1981. L'insertion sociale et professionnelle des jeunes. Rapport au Premier ministre. La Documentation française, Paris.

Stoffaës, C., 1995. Services publics. Questions d'avenir. Rapport, Commissariat général du plan. Odile Jacob, Paris.

Stoffaës, C. (Ed.), 1997. Services publics comparés en Europe. Exception française, exigence européenne. Séminaire de la promotion M. Bloch, tome I, ENA. La Documentation française, Paris.

Strobel, P., 1993. L'usager, le client et le citoyen. Quels rôles dans la modernisation du service public ? Recherches et prévisions 2, 31-44.

Sueur, J.P. (Ed.), 1998. Demain, la ville. Rapport au ministre de l'Emploi et de la solidarité. La Documentation française, Paris.

Tanter, A., Toubon, J.C., 1995. Vingt ans de politique française du logement social. Regards sur l'actualité 214, 30-50.

Tapie-Grime, M., 1997. Le Nimby, une ressource de démocratisation. Écologie politique 21, 13-26. 
Thin, D., et al., 1992. Processus de formation et dynamiques locales dans l'agglomération lyonnaise. Rapport de recherche, PPSH, GRS, université Lyon II. Thoenig, J.C., 1973. L'ère des technocrates. Le cas des Ponts et Chaussées. Éditions d'Organisation, Paris.

Thoenig, J.C., 1985. L'analyse des politiques publiques. In: Grawitz, M., Leca, J. (Eds.), Traité de science politique 4. Puf, Paris.

Topalov, C., 1987. Le logement en France. Histoire d'une marchandise impossible. Presses de la FNSP, Paris.

Toulemonde, B., 1998. Rapport de l'Inspection générale de l'Éducation nationale. Trom, D., 1999. De la réfutation de l'effet Nimby considéré comme une pratique militante. Notes pour une approche pragmatique de l'activité revendicative. Revue française de science politique 49 (1), 31-50.

Van Eeckhout, L. (Ed.), 1997. Vivre la ville demain. Quels enjeux ? Quels partenaires ? Séminaire de Cerisy-la-Salle, Collection rapports de recherche prospective et recherches sociétales 114, RATP, Paris.

Van Zanten, A., et al., 1991. Dynamiques urbaines et dynamiques scolaires. L'école comme enjeu de la recomposition d'un nouvel ordre urbain. Rapport de recherche, Plan urbain, Datar, Commissariat général du plan.

VEI (Ville-école-intégration), 2000. L'universel républicain à l'épreuve. Discrimination, ethnicisation, ségrégation (121).

VEI (Ville-école-intégration), 2002. Enseigner en milieu ethnicisé. Face à la discrimination. Actes du colloque du Réseau interculturel et éducation, mai 2002, hors-série 6.

Verdeil, V., 1998. L'équité territoriale. Espace géographique 3, 204-216.

Verpraet, G. (Ed.), 1997. Partenariat et politiques publiques dans les métropoles européennes. Séminaire européen, CNRS, PIR-Villes.

Warin, P., 1995. Contestations du service public et controverses sur les responsabilités de l'État. Le cas de l'entretien routier et des petits aménagements urbains par les services de l'équipement. Rapport de recherche, Cerat, ministère de l'Équipement 
Warin, P. (Ed.), 1997. Quelle modernisation des services publics? Les usagers au cœur des réformes. La Découverte, Paris.

Warin, P., 1999. La citoyenneté de guichet. Quelques éléments de définition et de discussion. In: Decreton, S. (Ed.), Service public et lien social. L'Harmattan, Paris.

Weber, Y., 1967. L'administration consultative. LGDJ, Paris.

Weller, J.M., 1993. Du trouble et de la transparence. Éléments d'observations d'une politique de communication. Recherches et prévisions 45-50.

Weller, J.M., 1998. La modernisation des services publics par l'usager. Une revue de la littérature (1986-1996). Sociologie du travail 40 (3), 365-392.

Wiel, M., et al., 1997. Comportements de mobilité et évolution de l'organisation urbaine. Université de Bretagne occidentale, Drast, Plan urbain, ministère de l'Équipement.

Wieviorka, M. (Ed.), 1991. Sociologie du racisme. Police et racisme. Rapport de recherche, Ihesi, ministère de l'Intérieur.

Wieviorka, M. (Ed.), 1992. La France raciste. Seuil, Paris.

Wieviorka, M. (Ed.), 1999. Violence en France. Seuil, Paris.

Wieviorka, M. (Ed.), 2002. La médiation. Une comparaison européenne. Les éditions de la DIV.

Worms, J.P., 1966. Le préfet et ses notables. Sociologie du travail 8 (3), 249-275. Wyvekens, A., 1998. Jeunesse en difficulté et justice de proximité. Rapport de recherche, CEPS, CEPEL, Mission de recherche « droit et justice », ministère de la Justice. 